\title{
Global geometry of regions and boundaries via skeletal and medial integrals
}

\author{
JAMES DAMON
}

For a compact region $\Omega$ in $\mathbb{R}^{n+1}$ with smooth generic boundary $\mathcal{B}$, the Blum medial axis $M$ is the locus of centers of spheres in $\Omega$ which are tangent to $\mathcal{B}$ at two or more points. The geometry of $\Omega$ is encoded by $M$, which is a Whitney-stratified set, and $U$, the multivalued vector field from points on $M$ to the points of tangency. We give general formulas for integrals of functions over $\mathcal{B}$ or $\Omega$ in terms of integrals over $M$. These integral formulas involve a radial shape operator which captures the radial geometry of $U$ on $M$, an intrinsic medial measure on $M$, and a radial flow from $M$ to $\mathcal{B}$. For integrals over $\Omega$, the formulas remain valid when we relax the conditions on $(M, U)$, yielding a more general skeletal structure. These integral formulas are applied to yield: an extension of Weyl's volume of tubes formula where we replace tubes by general regions; a medial version of the generalized GaussBonnet formula for $\mathcal{B}$, valid even for odd-dimensional $\mathcal{B}$; versions of Crofton-type formulas and Steiner formulas for subregions of $\Omega$ and a version of the divergence theorem over subregions in $\Omega$ for vector fields with discontinuities across the medial axis. This last result leads to a justification of an algorithm for finding the medial axis, using an invariant equivalent to a local medial density for singularities introduced elsewhere.

\section{Contents}

1. Introduction

2. Skeletal structures, shape operators and radial flow

3. Skeletal and medial integrals

4. Boundary integrals as medial integrals

\section{Medial version of the generalized Gauss-Bonnet} theorem 
6. Integrals over regions as skeletal integrals

7. Volumes of generalized tubes

8. Divergence theorem for fluxes with discontinuities across the medial axis

9. Computing the average outward flux for the grassfire flow

Acknowledgement

References

\section{Introduction}

We consider a compact $(n+1)$-dimensional region $\Omega \subset \mathbb{R}^{n+1}$ with smooth boundary $\mathcal{B}$. The geometry of both the region $\Omega$ and the boundary $\mathcal{B}$ is encoded by the Blum medial axis $M$ of $\Omega$ [1], which is the locus of centers of spheres in $\Omega$ tangent to $\mathcal{B}$ at two or more points allowing single degenerate tangencies as in figure 1.

For example, this medial axis plays a fundamental role in computer imaging for analyzing the shapes of objects [2]. $M$ can be alternately described as the Maxwell set for the family of distance functions on $\mathcal{B}[3]$, or the shock set for the "grassfire"/eikonal flow from $\mathcal{B}[4]$. As a result of these

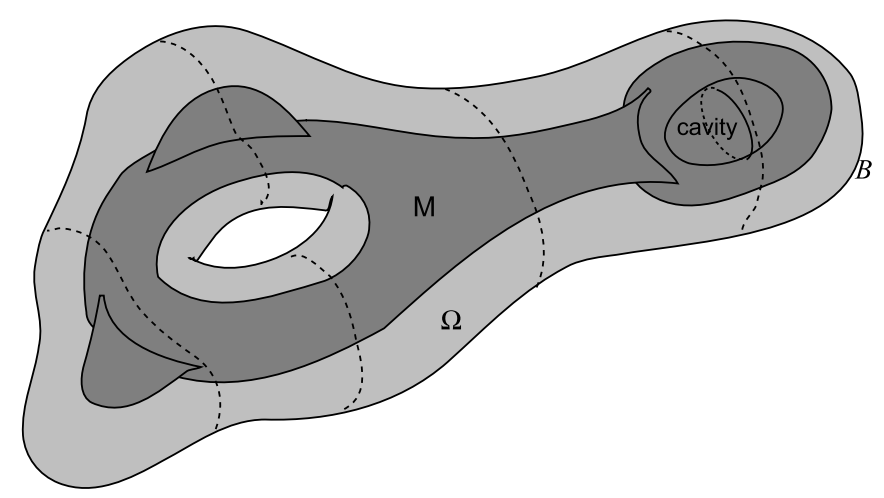

Figure 1: Blum medial axis $M$ of an object/region $\Omega$ with smooth boundary $\mathcal{B}$. 
multiple descriptions, the properties of $M$ are well understood for generic boundary $\mathcal{B} ; M$ is a Whitney-stratified set [3], whose local structure has been determined for $n \leq 6[3,5]$ (also see [25] and especially [6] for $n=2$ ).

On $M$ is defined a multivalued "radial vector field" $U$ from points on $M$ to the points of tangency of the spheres with the boundary (figure 2). In the earlier papers [7-9], we introduced geometric invariants of the "radial geometry" of $U$ on $M$ and used them to deduce both local and relative geometric properties of the boundary $\mathcal{B}$. In this paper, we determine how we may compute global geometric invariants of $\Omega$ and $\mathcal{B}$ in terms of integral invariants on $M$.

As an example, we recall that if $M$ is a closed smooth $n$-dimensional submanifold (without boundary) of $\mathbb{R}^{n+1}$, then the tube $T_{r}$ about $M$ of radius $r$ consists of points $\left\{x+t \mathbf{n}(x) \in \mathbb{R}^{n+1}: x \in M\right.$, and $\left.-r \leq t \leq r\right\}$, where $\mathbf{n}(x)$ is a unit normal vector to $M$ at $x$. For $r$ sufficiently small, $T_{r}$ is a manifold with boundary and $M$ is the Blum medial axis of $T_{r}$. The classical volume of tubes formula of Weyl [10] expresses the volume of a tube $T_{r}$ as a polynomial in $r$ whose coefficients are global curvature invariants. In the special case of the tube on the $n$-dimensional submanifold $M$ in $\mathbb{R}^{n+1}$, the formula has the form [11]

$$
\text { Volume of } T_{r}=(2 r) \cdot \sum_{j=0}^{[n / 2]} \frac{k_{2 j}(M) \cdot r^{2 j}}{1 \cdot 3 \cdot 5 \cdots(2 j+1)}
$$

where the terms $k_{2 j}(M)$ are integrals over $M$ of specific expressions in the curvature of $M$.

In this paper, we will generalize this result for general regions $\Omega$ with generic smooth boundaries $\mathcal{B}$. Moreover, rather than just computing the volume of $\Omega$, we will quite generally compute global geometric invariants of both $\Omega$ and $\mathcal{B}$ in terms of integrals over the Blum medial axis $M$. This will include the $n$-dimensional volume of $\mathcal{B}$, the $(n+1)$-dimensional volume of $\Omega$, the total curvature of $\mathcal{B}$, etc. We do this by deriving formulas for integrals of functions over $\Omega$ and $\mathcal{B}$ in terms of "skeletal integrals" over $M$ (where we relax several of the Blum conditions), and more specifically "medial integrals" when $M$ is a Blum medial axis.

In fact, we will express integrals over regions in $\Omega$ and $\mathcal{B}$ with piecewise smooth boundaries as integrals over corresponding regions in $M$. This leads to generalizations of Weyl's volume of tube formula to "generalized tubes" where $r$ is allowed to vary. These generalizations include: "generalized partial/half-tubes" defined using regions on $M$, versions of Steiner's formula for generalized offset regions, tubes allowing a singular boundary. 
As a second application of such formulas, we obtain a medial version of the Gauss-Bonnet theorem for $\mathcal{B}$ which is valid for all dimensions (including the case when the dimension of $\mathcal{B}$ is odd). Third, we derive a version of Gauss's theorem for flux integrals over regions of $\Omega$ where the vector field has discontinuities across $M$.

There are several surprising aspects of the formulas we obtain. The first is that unlike Weyl's formula, we do not directly involve the differential geometry of $M$. Instead we use expressions involving the "radial curvature" of the multivalued radial vector field $U$ on $M$. The second is that rather than involving integrals over $M$, the formulas involve integrals over $\tilde{M}$, the "double" of $M$, which is equivalent to integrating over "both sides of $M$ ". A third is that there is a natural "medial measure" on $\tilde{M}$, which takes into account the failure of $U$ to be normal to $M$ and replaces the usual Riemannian volume of $M$. This mathematical measure provides a heuristic measure of significance for various parts of the Blum medial axis, based on their contributions to the global geometric invariants of $\mathcal{B}$ and $\Omega$.

The basis for this approach is the introduction of a more general notion of a "skeletal structure" $(M, U)$ as a generalization of the Blum medial axis $M$. It consists of a special type of Whitney-stratified set $M$ on which is defined a multivalued radial vector field $U$ (figure 2). It has an associated boundary $\mathcal{B}$ which need not be smooth (conditions for the smoothness of $\mathcal{B}$ are derived in [7]).

In [7], we introduced for the skeletal structure $(M, U)$, a radial shape operator $S_{\text {rad }}$ (along with an edge shape operator $S_{\mathrm{E}}$ which will not be used in the integral formulas), and a compatibility 1 -form $\eta_{U}$. We may write $U=r \cdot U_{1}$ for a unit vector field $U_{1}$ and radius function $r$. Then, the radial shape operator $S_{\text {rad }}$ measures how $U_{1}$ varies on $M$. In [8, 9] we used these shape operators to compute the local differential geometric properties of the associated boundary $\mathcal{B}$ in the partial Blum case (this only requires one of the conditions which $M$ must satisfy to be a Blum medial axis; namely, that the radial vector is orthogonal to the boundary, which is guaranteed by a

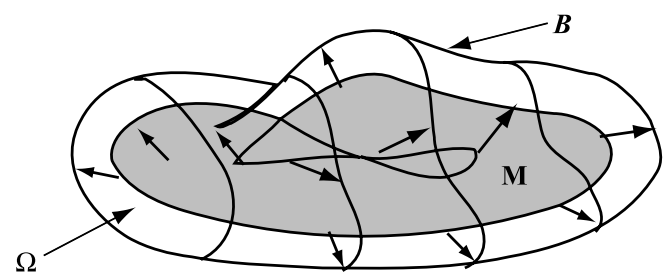

Figure 2: A skeletal structure $(M, U)$ defining a region $\Omega$ with smooth boundary $\mathcal{B}$. 
"compatibility condition" involving $\left.\eta_{U}\right)$. To relate geometrical information on the boundary with medial data, we introduced the "radial flow" from the skeletal set $M$ to the associated boundary $\mathcal{B}$, which is a backwards version of the "grassfire flow". Its associated time one map is the radial map $\psi_{1}$.

These same data will be used to express global geometric properties of the region and the boundary via "medial integrals" on the medial axis (or more generally "skeletal integrals" in the non-Blum case). First, in the partial Blum case, we generally express an integral of a function $g$ on the boundary as a medial integral of the associated multivalued function $\tilde{g}$ on the medial axis obtained by composing $g$ with the radial map $\psi_{1}$ (Theorem 4.1).

$$
\int_{\mathcal{B}} g d V=\int_{\tilde{M}} \tilde{g} \cdot \operatorname{det}\left(I-r S_{\mathrm{rad}}\right) d M,
$$

where the RHS is an integral over $\tilde{M}$, the "double" of $M$, which as already mentioned is equivalent to integrating over both sides of $M$. Also, the measure $d M$ is obtained by multiplying the volume form $d V$ on $M$ by a factor $\rho$ measuring the non-normality of $U$.

Second, applying the formula to the constant function 1 on $\mathcal{B}$ yields a medial integral formula for the $n$-dimensional volume of $\mathcal{B}$.

$$
n \text {-dimensional volume of } \mathcal{B}=\int_{\tilde{M}} \operatorname{det}\left(I-r S_{\text {rad }}\right) d M
$$

Third, we apply this medial integral formula to the medial integral of the radial curvature on the medial axis to obtain a Medial version of the generalized Gauss-Bonnet formula valid for $\mathcal{B}$ of all dimensions. This formula computes the classical Gauss-Bonnet integral of the Lipschitz-Killing curvature $K$ on $\mathcal{B}$ by a medial integral of the Radial Curvature $K_{\text {rad }}$ on the Blum medial axis $M$ (Theorem 5.3).

$$
\frac{1}{s_{n}} \cdot \int_{\mathcal{B}} K d V=\frac{1}{s_{n}} \cdot \int_{\tilde{M}} K_{\mathrm{rad}} d M=\chi(\Omega)=\chi(M)
$$

and if $n$ is even

$$
=\frac{1}{2} \chi(\mathcal{B})
$$

where $s_{n}=\operatorname{vol}\left(S^{n}\right)$ and $\chi(X)$ denotes the Euler characteristic of $X$.

Next, we can likewise compute integrals of functions $g$ over the entire region $\Omega$ in terms of "skeletal integrals", which are integrals over the skeletal set $M$ where we do not even require a partial Blum condition. If $\tilde{\psi}$ denotes 
the radial flow from $\tilde{M}$, we let $g_{1}(x, t)=g \circ \tilde{\psi}(x, t)$ (see Section 2 or [7, Section 4]). We let

$$
\tilde{g}=\int_{0}^{1} g_{1} \cdot \operatorname{det}\left(I-\operatorname{tr} S_{\mathrm{rad}}\right) d t
$$

Then, $\tilde{g}$ is a multivalued function on $M$. Hence, the integral of the product $\tilde{g} \cdot r$ is defined on $M$. We compute the integral of $g$ over $\Omega$ by the following integral over $\tilde{M}$ (Theorem 4.3).

$$
\int_{\Omega} g d V=\int_{\tilde{M}} \tilde{g} \cdot r d M
$$

In the special case that $g \equiv 1$, we let $\delta=\int_{0}^{1} \operatorname{det}\left(I-\operatorname{tr} S_{\text {rad }}\right) d t$. Then, we may compute the volume of $\Omega$ as a skeletal integral

$$
(n+1) \text {-dimensional volume of } \Omega=\int_{\tilde{M}} \delta \cdot r d M
$$

We furthermore derive versions of all of these formulas for integrals over regions of $\mathcal{B}$ (Corollary 4.2) or $\Omega$ (yielding a Crofton-type formula, Corollary 6.3). These versions allow us to obtain as corollaries formulas for integrals over and volumes of generalized, partial or singular tubes, including halftubes and generalized offset regions. We determine the forms of Weyl-type expansions of these integrals in terms of moment integrals along radial lines and "weighted medial and skeletal integrals" involving powers of $r$.

One consequence for computer imaging is that for 2-dimensional objects in $\mathbb{R}^{2}$ or 3 -dimensional objects in $\mathbb{R}^{3}$ with generic boundaries, global geometric quantities computed as either integrals over the whole region or on the boundary of the object can alternately be expressed as appropriate medial integrals on the Blum medial axis. This provides a framework for statisically analyzing properties of similar objects as in medial imaging [12], but now using medial data naturally weighted by the terms appearing in the integrals.

Finally, we derive a modified form of the divergence theorem for regions $\Gamma$ of $\Omega$ when vector fields exhibit discontinuities across the skeletal set (or medial axis) $M$ (Theorem 8.3). This modification requires a correction term involving medial or skeletal integrals. We apply this version to the vector field generating the grassfire flow (Theorem 8.5) to give in Section 9 a rigorous computation of the average outward flux for the grassfire flow, which justifies the algorithm given in [13] for numerically computing the Blum 
medial axis. In the course of this computation, we are led to introduce a medial density which in certain cases is surprisingly similar to a local density for singular varieties introduced in [14].

The author would like to especially thank both Mike Kerchove and Stephen Pizer for their valuable comments regarding this work, and to Kaleem Siddiqi for discussions concerning the role of flux integrals for the grassfire flow in $[13,15]$.

\section{Skeletal structures, shape operators and radial flow}

\subsection{Skeletal Structures}

We begin by recalling the definition of a skeletal structure $(M, U)$ in $\mathbb{R}^{n+1}$. Here $M$ is a skeletal set which is a special type of Whitney-stratified set. Hence, it which may be represented as a union of disjoint smooth strata $M_{\alpha}$ satisying the axiom of the frontier: if $M_{\beta} \cap \bar{M}_{\alpha} \neq \emptyset$, then $M_{\beta} \subset \bar{M}_{\alpha}$; and Whitney's conditions (a) and (b) (which involve limiting properties of tangent planes and secant lines). For example, for generic boundaries, the Blum medial axis is a Whitney-stratified set (by results of Mather [3] on the distance to the boundary function together with basic properties of Whitney-stratified sets, see, e.g., [16] or [17]). Additionally, $M$ may be locally decomposed into a union of $n$-dimensional manifolds $M_{j}$ with boundaries and corners which only intersect on boundary faces.

We let $M_{\text {reg }}$ denote the points in the top-dimensional strata (this is the dimension $n$ of $M$ and these points are the "smooth points" of $M$ ). Also, we let $M_{\text {sing }}$ denote the union of the remaining strata. On $M$ is defined a multivalued vector field $U$, which is called the radial vector field. For a regular point $x \in M$, there are two values of $U$. For each value of $U$ at $x$, there are choices of values at neighboring points which form a smooth vector field on a neighborhood of $x$. Moreover, $U$ satisfies additional conditions at edge points of $M$ and singular points of $M$, see [7, Section 1] for more details.

For a radial vector field $U$, we may represent $U=r \cdot U_{1}$, for a positive multivalued function $r$, and a multivalued unit vector field $U_{1}$ on $M$. These satisfy analogous properties to $U$.

\subsection{Radial-shape operator}

For the full understanding of the geometry of the boundary, two shape operators are needed, the radial and edge-shape operators. However, because edge-shape operators are only needed on a set of measure zero, we will be 
able to ignore them when considering integrals. For a regular point $x_{0}$ of $M$ and each smooth value of $U$ defined in a neighborhood of $x_{0}$, with associated unit vector field $U_{1}$, the radial-shape operator is defined by

$$
S_{\mathrm{rad}}(v)=-\operatorname{proj}_{U}\left(\frac{\partial U_{1}}{\partial v}\right)
$$

for $v \in T_{x_{0}} M$. Here $\operatorname{proj}_{U}$ denotes projection onto $T_{x_{0}} M$ along $U$ (which in general is not orthogonal to $T_{x_{0}} M$ ). Because $U_{1}$ is not necessarily normal and the projection is not orthogonal, it does not follow that $S_{\text {rad }}$ is selfadjoint as is the case for the usual differential geometric shape operator. We let $K_{\mathrm{rad}}=\operatorname{det}\left(S_{\mathrm{rad}}\right)$ and refer to it as the radial curvature.

For a point $x_{0}$ and a given smooth value of $U$, we call the eigenvalues of the associated operator $S_{\text {rad }}$ the principal radial curvatures at $x_{0}$, and denote them by $\kappa_{r i}$. As $U$ is multivalued, so are $S_{\mathrm{rad}}$ and $\kappa_{r i}$.

2.2.1. Compatibility 1-forms. To identify the partial Blum condition for the boundary, we use the compatibility 1-form. Given a smooth value for $U$, (possibly at a point of $M_{\text {sing }}$ ), with $U=r \cdot U_{1}$ for a unit vector field $U_{1}$, we define the compatibility 1 -form $\eta_{U}(v) \stackrel{\text { def }}{=} v \cdot U_{1}+d r(v)$. This is a multivalued 1 -form. The vanishing of $\eta_{U}$ at $x_{0}$ implies that $U\left(x_{0}\right)$ is orthogonal to the tangent space of the associated boundary $\mathcal{B}$ at the corresponding point $[7$, Lemma 6.1].

In [7, Theorem 1] we give three conditions: radial curvature condition, edge condition and compatibility condition, which together ensure that the associated boundary of the skeletal structure is smooth. These conditions are satisfied by the Blum medial axis in the generic case. We assume throughout the rest of this paper that these conditions are satisfied. Then, integrals are defined on $\mathcal{B}$. We will relate them to integrals on the skeletal set $M$.

\subsection{Radial flow and tubular neighborhood for a skeletal structure}

We stated in the introduction that in the partial Blum case we relate the geometry of boundary to the radial geometry of the skeletal set via the radial flow. One way to view the formation of the medial axis is as the shock set resulting from the Grassfire/level-set flow from the boundary [4] (see figure $3 \mathrm{~b}$ ); $[2,15]$ also see for further discussion. This flow is from points on the boundary along the normals until shocks are encountered. 

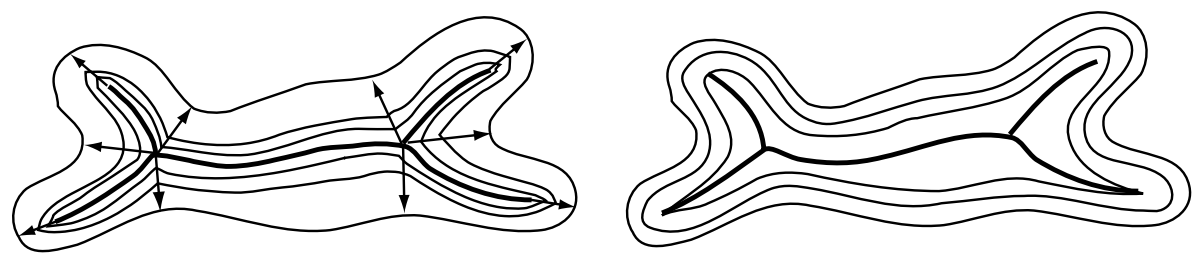

Figure 3: (a) Radial flow and (b) grassfire flow.

We would like to define the radial flow as essentially a "backward flow" along $U$ to relate the skeletal set $M$ with the boundary $\mathcal{B}$. Locally if we choose a smooth value of $U$ defined on a neighborhood $W$ of $x_{0} \in M$, we can define a local radial flow $\psi(x, t)=x+t \cdot U(x)$ on $W \times I$. We cannot use such local radial flows to define a global one on $M$ because the radial vector field $U$ is multivalued on $M$. We overcome this problem by introducing "double" $\tilde{M}$ of $M$ on which is defined a "normal bundle" $N$ for $(M, U)$.

\subsubsection{The double and the normal bundle of $M$ and the global} radial flow. Points of $\tilde{M}$ consist of pairs $\left(x, U^{\prime}\right)$ with $x \in M$ and $U^{\prime}$ a value of $U$ at $x$. It is possible to put the structure of a stratified set on $\tilde{M}$ so the natural projection $p: \tilde{M} \rightarrow M$ sending $\left(x, U^{\prime}\right) \mapsto x$ is continuous and smooth on strata. Moreover, on $\tilde{M}$, we have a canonical line bundle $N$ which at a point $\left(x_{0}, U_{0}\right)$ is spanned by $U_{0}$. Also, given an $\varepsilon>0$ we have the positive $\varepsilon$ neighborhood of the zero section $N_{\varepsilon}=\left\{\left(x_{0}, t U_{0}\right) \in N: 0 \leq t \leq \varepsilon\right\}$.

Now, for $(M, U)$, with normal line bundle $N$, we can define the global radial flow as a map $\tilde{\psi}: N \rightarrow \mathbb{R}^{n+1}$ by $\left(x_{0}, t U_{0}\right) \mapsto x_{0}+t U_{0}$. We proved in [7] that there is a sufficiently small $\varepsilon>0$ so that $\tilde{\psi} \mid N_{\varepsilon} \backslash \tilde{M}$ is a homeomorphism, smooth on regular points, and $M$ together with $\tilde{\psi}\left(N_{\varepsilon} \backslash \tilde{M}\right)$ forms a "tubular neighborhood" of $M[7$, Theorem 5.1].

We computed in [7] the derivatives of the radial flow $\tilde{\psi}$ and the radial map $\psi_{1}$. If $\mathbf{v}=\left\{v_{1}, \ldots, v_{n}\right\}$ is a basis for $T_{x_{0}} M$, we use bases $\left\{\partial / \partial t, v_{1}, \ldots, v_{n}\right\}$ in the source for $T_{x_{0}} M \times \mathbb{R}$ at $\left(x_{0}, t\right)$ and $\left\{U_{1}, v_{1}, \ldots, v_{n}\right\}$ for $\mathbb{R}^{n+1}$ at $\psi\left(x_{0}, t\right)$ by translation along $U$. Then the transpose of the Jacobian matrix of the radial flow with respect to these bases is equation (4.5) of [7].

$$
\left(\begin{array}{cc}
r & 0 \\
t\left(d r(\mathbf{v})+r A_{\mathbf{v}}\right) & \left(I-t r \cdot S_{\mathbf{v}}\right)^{\mathrm{T}}
\end{array}\right),
$$

where $S_{\mathbf{v}}$ denotes a matrix representation of $S_{\text {rad }}$ with respect to the basis $\mathbf{v}$.

Likewise, the matrix representation of the Jacobian of $\psi_{1}$ using the basis $\mathbf{v}$ for $T_{x_{0}} M$ and the same basis for $\left\{U_{1}, v_{1}, \ldots, v_{n}\right\}$ for $\mathbb{R}^{n+1}$ has tranpose 
given by deleting the first row of (2.1) to yield

$$
\left(\left(d r(\mathbf{v})+r A_{\mathbf{v}}\right) \quad\left(I-r \cdot S_{\mathbf{v}}\right)^{\mathrm{T}}\right) .
$$

The conditions that these maps are non-singular follow from the radial curvature condition:

$$
r<\min \left\{\frac{1}{\kappa_{r i}}\right\} \quad \text { for all positive principal radial curvatures } \kappa_{r i},
$$

where $\kappa_{r i}$, the "principal radial curvatures", are the eigenvalues of $S_{\mathrm{rad}}$ (there is an analogous edge condition on the "principal edge curvatures" $\kappa_{E} i$, which are generalized eigenvalues of $S_{\mathrm{E}}$ ).

The radial and edge conditions together ensure that the radial flow remains non-singular from smooth points and does not develop new singularities from singular points of $M$. These conditions: together with a compatibility condition involving the vanishing of the compatibility 1-form $\eta_{U}$ on $M_{\text {sing }}$ are (necessary and) sufficient to ensure that the "associated boundary" $\mathcal{B}$ is smooth (in the sense of [7, Theorem 2.5]) provided the radial map has no non-local self-intersections.

Definition 2.1. Suppose $(M, U)$ is a skeletal structure which satisfies: the radial curvature, edge conditions and compatibility conditions, and for which the radial flow does not have any non-local intersections. Then, we say that $(M, U)$ defines a region with smooth boundary.

If in addition, $U$ is everywhere orthogonal to the associated boundary $\mathcal{B}$, which is equivalent to the vanishing of the compatibility 1 -form $\eta_{U}$ on all of $M$, we say that $(M, U)$ satisfies the partial Blum condition.

If we relax the radial curvature and edge conditions by replacing the inequalities " $<$ " by " $\leq$ ", but suppose that the radial flow is still one-one for $0<t<1$, then we say that $(M, U)$ defines a region with (possibly) singular boundary $\mathcal{B}$.

For a Blum medial axis $M$ of a region $\Omega$ with generic smooth boundary $\mathcal{B}$ with its associated multivalued vector field $U,(M, U)$ is an example of a skeletal structure which satisfies the partial Blum condition and defines a region with smooth boundary.

Remark 2.2. In the case when $(M, U)$ "defines a region with smooth boundary", we know that $\mathcal{B}$ is smooth off the image $\psi\left(M_{\text {sing }}\right)$ of the singular set of $M$, where we only know $\mathcal{B}$ is weakly $C^{1}$. The images of the 
strata of $M_{\text {sing }}$ are still smooth submanifolds in $\mathcal{B}$. Then, $\mathcal{B}$ is piecewise smooth and so has a Riemannian volume form, denoted by $d V$, for each compact piecewise smooth region. Hence, integrals of continuous functions are defined on $\mathcal{B}$.

We next turn to defining integrals on $M$ and relating them to integrals on $\mathcal{B}$ and $\Omega$.

\section{Skeletal and medial integrals}

Given a skeletal structure $(M, U)$ which defines a region with smooth boundary, and a multivalued function $h$ on $M$, we will introduce the skeletal integral of $h$ over $M$. By a multivalued function $h$ on $M$, we mean a function which lifts to a well-defined function $h^{\prime}$ on $\tilde{M}$. Alternately, it means that for each value of $U$ at a point $x \in M$, there is a corresponding value of $h$ at $x$ and conversely. We say $h$ is a continuous multivalued function if $h^{\prime}$ is continuous on $\tilde{M}$. For example, if $g: \mathcal{B} \rightarrow \mathbb{R}$ is a continuous function on $\mathcal{B}$, then the composition $g \circ \psi_{1}$ defines a continuous function on $\tilde{M}$ which pushes down to a continuous multivalued function $\tilde{g}$ on $M$.

Our strategy will be to first define a skeletal integral for continuous multivalued continuous functions on $M$. This will be equivalent to defining the integral of a continuous function on $\tilde{M}$. This integral will satisfy the usual linearity and positivity properties. Then, as $\tilde{M}$ is a locally compact Hausdorff space, we shall use the Riesz representation theorem to extend the definition to general integrable functions over measurable regions of $\tilde{M}$. These will include, for example, piecewise continuous functions over regions of $\tilde{M}$ with piecewise differentiable boundaries.

By the compactness of $M$ and the properties of skeletal structures, we may find a finite covering of $M$ by open sets $\left\{W_{i}\right\}$ which satisfy the following properties: (i) for each $W_{i}$, and each stratum $M_{\alpha}$ for which $W_{i \alpha}=W_{i} \cap$ $M_{\alpha} \neq \emptyset$, each value of $U$ defined at a point of $W_{i \alpha}$ extends smoothly to values of $U$ on all of $W_{i \alpha}$; (ii) the closure of each $W_{i}$ may be decomposed into a finite number of manifolds with boundaries and corners which only meet along boundary facets. We refer to such a $W_{i}$ as a paved neighborhood (figure 4). If $\pi: \tilde{M} \rightarrow M$ denotes the natural projection, and $\pi^{-1}\left(W_{i}\right)=\cup W_{i j}$ is a disjoint union of neighborhoods in $\tilde{M}$, then we also refer to each $W_{i j}$ as a paved neighborhood of $\tilde{M}$. Each $W_{i j}$ is a union of manifolds with boundaries and corners on which values of $U$ can be chosen to form a continuous vector field.

Then, we may construct a smooth partition of unity $\left\{\chi_{i}\right\}$ subordinate to $\left\{W_{i}\right\}$. By smooth we mean that each $\chi_{i}$ is smooth on each stratum (we can 
in fact extend $\left\{W_{i}\right\}$ to an open covering $\left\{W_{i}^{\prime}\right\}$ of $M$ in $\mathbb{R}^{n+1}$ and restrict the corresponding partition of unity to $M)$. Then, using $\left\{\chi_{i}\right\}$ we define

$$
\int_{\tilde{M}} h d M=\sum_{i} \int_{\tilde{M}} \chi_{i} \cdot h d M .
$$

By a standard argument, (3.1) is independent of the covering and partition of unity. Thus, by (3.1), it will be sufficient to define the integral for $h$ with support in a finite union of compact manifolds with boundaries and corners $\left\{M_{j}\right\}_{j=1}^{s}$ which only meet along boundary facets as in figure 4 . In turn, for such an $h$ with $\operatorname{supp}(h) \subset \cup_{j=1}^{s} M_{j}$, we may write

$$
\int_{\tilde{M}} h d M=\sum_{i} \int_{\tilde{M}_{i}} h d M .
$$

Here $\tilde{M}_{i}$ is the union in $\tilde{M}$ of the two copies of $M_{i}$ corresponding to the two choices of smooth values of $U$ on $M_{i}$, which, in turn, correspond to the two sides of $M$ at $M_{i}$.

Remark 3.1. In the generic case where the Blum medial axis is the Maxwell set for multi- $A_{k}$ singularities, there are unique limiting tangent spaces for strata and we can decompose neighborhoods into manifolds with boundaries and corners as indicated. This always holds for $n \leq 6$. Whether this holds in general is unknown, but there is some evidence that this is true. If not, then the parametrization by manifolds with boundaries and corners may be singular on the boundary points which map to the singular set of $M$ (see [26] or [28] and [27]). Because the radial flow will be non-singular off the singular set for the Blum medial axis [7, Proposition 4.6], the change of variables formula will be valid, and the integrals are well defined.

Also, at edge points of the medial axis, we must use "edge coordinates". Under these, the parametrization of a neighborhood of an edge is one-one

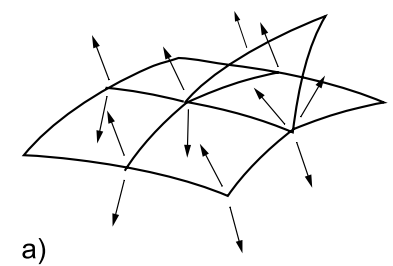

a)

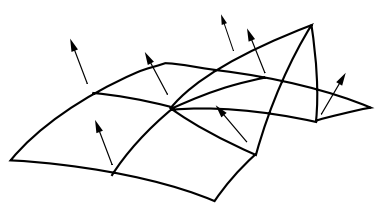

b)

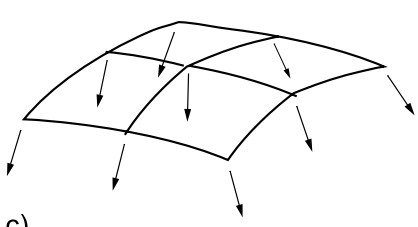

c)

Figure 4: (a) Paved neighborhood of a point in $M$ and corresponding paved neighborhoods (b) and (c) in $\tilde{M}$. 
differentiable and a local diffeomorphism off the edge. Again, this does not cause any problem with integration.

To finally define each integral on the RHS of (3.2), we let $j=1,2$ correspond to a value of $U$ on each side of $M_{i}$. We also let $h_{j}$ be the value of $h$ corresponding to that side, and let $\rho_{j}=U_{1 j} \cdot \mathbf{n}_{j}$, where $U_{1 j}$ is a value of the unit vector field for each of the smooth values of $U$ and $\mathbf{n}_{j}$ is the normal unit vector pointing on the same side as $U_{1 j}$. Lastly, we let $d V_{j}$ denote the volume form for the Riemannian metric with $M_{i}$ oriented by $\mathbf{n}_{j}$. Hence, we may finally write

$$
\int_{\tilde{M}_{i}} h d M=\sum_{j=1}^{2} \int_{M_{i}} h_{j} \rho_{j} d V_{j} .
$$

Then, the integral has the usual properties that it is linear; and if $h \geq 0$, then $\int h d M \geq 0$.

Next, we conclude that there is a unique regular Borel measure on $M$ such that the integral we just defined is given by integration with respect to this measure.

Proposition 3.2. There is a unique regular positive Borel measure $d M$ on $\tilde{M}$ such that for any continuous multivalued function $h$ on $M$ with $\tilde{h}=h \circ \pi$, the integral of $h$ on $\tilde{M}$ is given by

$$
\int_{\tilde{M}} \tilde{h} d M
$$

the integral of $\tilde{h}$ with respect to the measure $d M$.

Proof. As stated earlier, we use the Riesz Representation Theorem to prove the existence and uniquence of $d M$. For this we have already noted that we can view the integral as being defined for continuous functions $\tilde{h}$ on $\tilde{M}$. Then, we can extend this integral to (compactly supported) complex-valued continuous functions $f=g+i h$ on $\tilde{M}$ by

$$
\int_{\tilde{M}} f d M=\int_{\tilde{M}} g d M+i \cdot \int_{\tilde{M}} h d M .
$$

This defines a linear transformation

$$
\int_{\tilde{M}} f d M: C_{c}(\tilde{M}) \longrightarrow \mathbb{C}
$$


for $C_{c}(\tilde{M})$ the space of (compactly supported) continuous complex-valued functions on $\tilde{M}$. This integral satisfies the positivity condition: $f \geq 0$ implies $\int_{\tilde{M}} f d M \geq 0$.

Then, $M \subset \mathbb{R}^{n+1}$ is closed (as it is compact) and hence locally compact as $\mathbb{R}^{n+1}$ is. We easily see that $\tilde{M}$ is also locally compact and Hausdorff (as well as compact). Thus, we may apply the Riesz representation theorem [18, Theorem 2.14]. There is a unique positive Borel measure $d M$ on $\tilde{M}$ (which is regular as $\tilde{M}$ is compact) defined on a $\sigma$-algebra of subsets of $\tilde{M}$ which contains the Borel sets such that $\int_{\tilde{M}} h d M$ is given by integration with respect to the measure $d M$ for any continuous $h$ on $\tilde{M}$. This is the asserted measure.

\subsection{Medial measure and Blum medial axis $M$ as a measure space}

We refer to the measure $d M=\rho d V$ on $\tilde{M}$ as the medial measure. It corrects for the failure of $U$ to be orthogonal to $M$. In the case of the Blum medial axis, $d M$ is actually defined on $M$.

This changes our perspective on the Blum medial axis from just being a stratified set to being as well a measure space, where the significance of parts of the space are determined by their medial measure. For example, it is well known that the introduction of a small bump on the boundary $\mathcal{B}$ leads to the creation of another sheet of $M$. However, the bump only introduces a small change in the volume. We shall see from the volume formulas in Section 6 that the additional integral on the added sheet represents this small change. The smallness of the bump forces $U$ to be close to being tangent to $M$ on the additional sheet. This implies that the medial measure is small on the added sheet. Thus, although set theoretically the added sheet is a significant alteration of the medial axis, from the point of view of measure theory the added sheet is very small.

\subsection{Skeletal and medial integrals}

By a multivalued measurable, resp., integrable, functon $f$ on $M$ we mean that $f \circ \pi$ is a measurable, resp., integrable, function on $\tilde{M}$, where $\pi: \tilde{M} \rightarrow$ $M$ is the natural projection. Likewise, we say that $R \subset \tilde{M}$ is measurable if it is measurable with respect to $d M$. If $R \subset \tilde{M}$ is measurable, and $f$ is defined on $R$, then provided $\chi_{R} \cdot f$ is measurable for the characteristic function $\chi_{R}$ of $R$, we define as usual $\int_{R} f d M=\int_{\tilde{M}} \chi_{R} \cdot f d M$. 
We refer to the integral $\int_{\tilde{M}} h d M$ as a skeletal integral. In the special case that $(M, U)$ satisfies the partial Blum condition, we will refer to it instead as a medial integral.

Measurable sets include regions with piecewise smooth boundary on $\tilde{M}$

Definition 3.3. A closed subset $R \in \tilde{M}$ is a region with piecewise smooth boundary if we can decompose $R=\cup_{i=1}^{\ell} R_{i}$ where: (i) the $R_{i}$ only intersect at boundary points; (ii) each $R_{i} \subset W_{i j}$, where $W_{i j}$ is a paved neighborhood in $\tilde{M}$; (iii) we may represent $W_{i j}$ as a finite union of manifolds with boundaries and corners $M_{\alpha}$ in $M$ so that $\pi\left(R_{i}\right) \cap M_{\alpha}$ is a region with piecewise smooth boundary

Heuristically, we view a region of $\tilde{M}$ as associating a region of a smooth stratum of $M$ to each side of $M$. For example, consider in figure 5 the region of $\tilde{M}$ consisting of points where at the corresponding points on $\mathcal{B}$, the Gaussian curvature is positive. It consists of the bottom side of $M$ and part of the top side as indicated in figure 5 .

Also, integrable functions include, for example, piecewise continuous functions

Definition 3.4. Let $g$ be a multivalued function on $M$. We say that $g$ is piecewise continuous if for $g^{\prime}=g \circ \pi, \operatorname{supp}\left(g^{\prime}\right)=\cup S_{j}$, where the $S_{i}$ only intersect at boundary points, each $S_{j}$ is a region with piecewise smooth boundary, and $\tilde{g} \mid \operatorname{int}\left(S_{j}\right)$ has a continuous extension to $S_{j}$.

If $g: \mathcal{B} \rightarrow \mathbb{R}$ is a piecewise continuous function on $\mathcal{B}$, then the composition $g \circ \psi_{1}$ need not define a piecewise continuous function on $\tilde{M}$, but it does define a Borel measurable one.

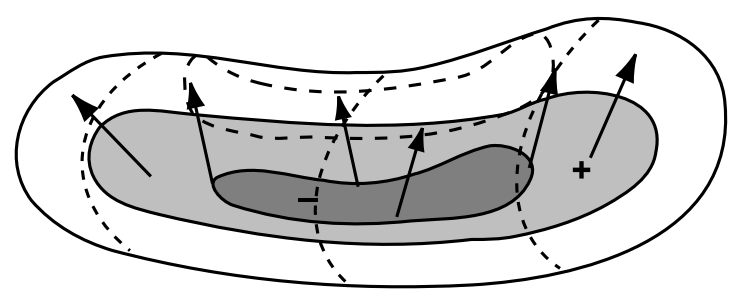

Figure 5: Region in $M$ where $\mathcal{B}$ has positive Gauss curvature. 


\section{Boundary integrals as medial integrals}

We now suppose that $(M, U)$ is a skeletal structure which defines a region with smooth boundary and satisfies the partial Blum condition. We know that $\mathcal{B}$ is smooth off the image $\psi_{1}\left(M_{\text {sing }}\right)$ of the singular set of $M$, where we only know it is weakly $C^{1}$. The images of the strata of $M_{\text {sing }}$ are still smooth submanifolds of $\mathcal{B}$, and using the radial map we see that points in $\psi_{1}\left(M_{\text {sing }}\right)$ have paved neighborhoods. Then, $\mathcal{B}$ is piecewise smooth and so has a Riemannian volume form, denoted by $d V$; hence, the same argument used for $M$ allows us to define the integral $\int_{\mathcal{B}} g d V$ for a continuous function $g$. Then, even if $\mathcal{B}$ is not smooth we can still use the Riesz representation theorem to extend the integral for Borel measurable functions and regions on $\mathcal{B}$.

Theorem 4.1. Suppose $(M, U)$ is a skeletal structure defining a region with smooth boundary $\mathcal{B}$ and satisfying the partial Blum condition. Let $g: \mathcal{B} \rightarrow \mathbb{R}$ be Borel measurable and integrable with respect to the Riemannian volume measure. Then,

$$
\int_{\mathcal{B}} g d V=\int_{\tilde{M}} \tilde{g} \cdot \operatorname{det}\left(I-r S_{\mathrm{rad}}\right) d M
$$

where $\tilde{g}=g \circ \psi_{1}$.

Before beginning the proof, we deduce several consequences.

First, we have a version for a region of $\mathcal{B}$.

Corollary 4.2. Suppose $(M, U)$ is a skeletal structure defining a region with smooth boundary $\mathcal{B}$ and satisfying the partial Blum condition. Let $R$ denote a Borel measurable subset of $\mathcal{B}$ and $g: R \rightarrow \mathbb{R}$ a Borel measurable and integrable function with respect to the Riemannian volume measure. If $\tilde{R}=\psi_{1}^{-1}(R)$, then,

$$
\int_{R} g d V=\int_{\tilde{R}} \tilde{g} \cdot \operatorname{det}\left(I-r S_{\mathrm{rad}}\right) d M,
$$

where $\tilde{g}=g \circ \psi_{1}$.

Proof of Corollary 4.2. If $\chi_{R}$ denote the characteristic function of $R$. Then, $\chi_{R} \cdot g$ is a Borel measurable and integrable function on $\mathcal{B}$. Thus, we may 
apply Theorem 4.1 to conclude

$$
\int_{\mathcal{B}} \chi_{R} \cdot g d V=\int_{\tilde{M}}\left(\chi_{R} \cdot g\right) \circ \psi_{1} \cdot \operatorname{det}\left(I-r S_{\mathrm{rad}}\right) d M .
$$

The LHS of equation (4.3) is $\int_{R} g d V$. Also, $\left(\chi_{R} \cdot g\right) \circ \psi_{1}=\chi_{\tilde{R}} \cdot \tilde{g}$. Thus, the RHS of Equation (4.3) is the RHS of Equation (4.2) as asserted.

As a first application, we compute the $n$-dimensional volume of $\mathcal{B}$ as a medial integral.

Theorem 4.3 (Medial Integral Formula for Boundary Volume). Suppose $\Omega \subset \mathbb{R}^{n+1}$ is a region with compact closure and smooth generic boundary $\mathcal{B}$ and Blum medial axis $M$. Then,

$$
n \text {-dimensional volume of } \mathcal{B}=\int_{\tilde{M}} \operatorname{det}\left(I-r S_{\mathrm{rad}}\right) d M
$$

Remark 4.4. The preceding formula remains true if $(M, U)$ is only a skeletal structure in $\mathbb{R}^{n+1}$ defining a region with smooth boundary $\mathcal{B}$ and satisfying the partial Blum condition. Also, it remains valid if we replace $\mathcal{B}$ by a measurable region $R$ and $\tilde{M}$ by $\tilde{R}$.

Proof. We apply Theorem 4.1 for the constant function 1 on $\mathcal{B}$. Even if $\mathcal{B}$ is only piecewise smooth, the integral of 1 over $\mathcal{B}$ still yields the $n$-dimensional volume of $\mathcal{B}$. On the other hand, by Theorem 4.1 , the integral equals the RHS of (4.4).

\subsection{Expansion of the boundary volume in terms of the radial function $r$}

We expand the integrand in the RHS of (4.1). Let $\sigma_{r j}$ denote the $j$ th elementary symmetric function in the principal radial curvatures $\kappa_{r i}$ (with $\left.\sigma_{r 0} \equiv 1\right)$. Then, we may expand

$$
\operatorname{det}\left(I-r S_{\mathrm{rad}}\right)=\sum_{i=0}^{n}(-1)^{i} \sigma_{r i} r^{i}
$$

Then, we can expand (4.1) using $\ell$-th weighted integrals of the multivalued function $g$ defined by

$$
I_{\ell}(g)=\int_{\tilde{M}} g \cdot r^{\ell} d M
$$


Then we may expand the RHS of (4.1)

$$
\int_{\mathcal{B}} g d V=\sum_{i=0}^{n}(-1)^{i} I_{i}\left(g \cdot \sigma_{r i}\right)
$$

In the case that $g \equiv 1$, we obtain an expansion for the volume of $\mathcal{B}$.

$$
n \text {-dimensional volume of } \mathcal{B}=\sum_{i=0}^{n}(-1)^{i} I_{i}\left(\sigma_{r i}\right) \text {. }
$$

In general, $r$ is not constant and cannot be taken outside the integral. If $r$ is constant, then the partial Blum condition implies that $U$ is normal at all points. Then, $M$ must be a closed submanifold without boundary. Thus, this is the case of a tube. As $M$ is smooth with $U$ normal, we have two consequences. First, the radial shape operator is the differential geometric shape operator. However, there is one for each side of $M$ at a point $x_{0}$, and the $U_{1}$ on one side is the negative of that on the other. Hence, the principal curvatures computed for each side differ by signs. Thus, the $\sigma_{r i}$ for each side differ by $(-1)^{i}$. Thus, the integrals of these on each side will cancel in the case $i$ is odd. Thus, we obtain a polynomial representation.

Corollary 4.5. The $n$-dimensional volume of the boundary $\mathcal{B}$ of the tube on $M$ of radius $r$ is given by

$$
n \text {-dimensional volume of } \mathcal{B}=\sum_{i=0}^{[n / 2]}\left(\int_{\tilde{M}} \sigma_{r 2 i} d M\right) \cdot r^{2 i}
$$

where now $\sigma_{r 2 i}$ is the $2 i$-th elementary symmetric function in the principal curvatures of $M$, and

$$
K_{j}=\int_{\tilde{M}} \sigma_{r j} d M
$$

is a global curvature invariant

Equation (4.8) gives a formula for the $n$-dimensional volume of $\mathcal{B}$, which is a union of two parallel manifolds. Without requiring the partial Blum condition, we have much greater flexibility in allowing a variety of generalizations of tubes. We will obtain generalizations of Weyl's formula for the volumes of such generalized tubes in Section 7. 
Example 4.6. We consider the special cases of $\Omega \subset \mathbb{R}^{2}$ or $\mathbb{R}^{3}$. In the first case, Theorem 4.3 via (4.8) gives a formula for the length of the boundary curve $\mathcal{B}$. There is a single radial curvature $\kappa_{r}$. Then,

$$
\begin{aligned}
\text { length }(\mathcal{B}) & =\int_{\tilde{M}} 1-r \kappa_{r} d M \\
& =\int_{\tilde{M}} d M-\int_{\tilde{M}} r \kappa_{r} d M
\end{aligned}
$$

The first integral on the RHS of (4.10) is $2 \tilde{\ell}(M)$, where $\tilde{\ell}(M)$ is the length of $M$, but with respect to the "medial Riemannian length" $d M=d \tilde{s}=\rho \cdot d s$.

For the second case of $\Omega \subset \mathbb{R}^{3}$, we have

$$
\operatorname{det}\left(I-r S_{\mathrm{rad}}\right)=1-r \cdot \operatorname{trace}\left(S_{\mathrm{rad}}\right)+r^{2} \operatorname{det}\left(S_{\mathrm{rad}}\right)
$$

Hence, for the surface $\mathcal{B}$, letting $H_{\text {rad }}=1 / 2 \operatorname{trace}\left(S_{\text {rad }}\right)$ and $K_{\text {rad }}=\operatorname{det}\left(S_{\text {rad }}\right)$, we obtain

$$
\operatorname{area}(\mathcal{B})=\int_{\tilde{M}} d M-2 \int_{\tilde{M}} r H_{\text {rad }} d M+\int_{\tilde{M}} r^{2} K_{\text {rad }} d M
$$

Again the first integral on the RHS represents twice the area of $M$ but measured using the "medial Riemannian area form" $\tilde{d} A=\rho \cdot d A$.

Lastly, we turn to the proof of Theorem 4.1.

Proof. By the proof of Theorem 5.1 of [7] $\psi_{1}: \tilde{M} \rightarrow \mathcal{B}$ is a homeomorphism. Hence, we have the pull-back $\psi_{1}^{*}(d V)$ defined on $\tilde{M}$. Also, by general properties, for a Borel measurable function $g$ on $\mathcal{B}$,

$$
\int_{\tilde{M}} \psi_{1}^{*} g \psi_{1}^{*} d V=\int_{\mathcal{B}} g d V
$$

Hence, Theorem 4.1 will follow provided we can show $\psi_{1}^{*} d V=d M$. Again, by the uniqueness of the measure in the Riesz representation theorem, this will follow if

$$
\int_{\tilde{M}} h \psi_{1}^{*} d V=\int_{\tilde{M}} h d M
$$

for continuous functions $h$ on $\tilde{M}$.

As any $h=\psi_{1}^{*} g$ for $g=\psi_{1}^{-1 *} h$, it is enough to establish the theorem for continuous $g$. Using a partition of unity argument as in the definition of skeletal integrals in Section 3, we may assume $\psi_{1}^{*} g$ has support in a single 
compact manifold with boundaries and corners $M_{i}$ whose interior consists of regular points of $M$, and for a single smooth value of $U$ defined on $M_{i}$. For then, by the form of the integrals given in Section 3, we can sum both sides over such integrals to obtain equality for all of $M$.

Let $\mathcal{B}_{i}=\psi_{1}\left(M_{i}\right)$. As $\psi_{1}: M_{i} \rightarrow \mathcal{B}_{i}$ is the restiction of a diffeomorphism, we can use the change of variables formula. On $\mathcal{B}_{i}$,

$$
d V\left(w_{1}, \ldots, w_{n}\right)=\operatorname{det}\left(\mathbf{n}^{\prime}, w_{1}, \ldots, w_{n}\right),
$$

where $\mathbf{n}^{\prime}$ denotes the outward pointing unit normal vector. By the (partial) Blum condition, $\mathbf{n}_{\psi_{1}(x)}^{\prime}=U_{1}(x)$, the unit vector in the direction of $U$.

Next, by earlier calculations in (2.2) [7, Section 2], if $\left\{v_{1}, \ldots, v_{n}\right\}$ denotes a basis for $T_{x} M_{i}$, then

$$
\frac{\partial \psi_{1}}{\partial v_{i}}=b_{i} \cdot U_{1}-\sum_{j=1}^{n} c_{j i} v_{j},
$$

where the matrix $C=\left(c_{i j}\right)=\left(I-r \cdot S_{\mathbf{v}}\right)^{\mathrm{T}}$, and $S_{\mathbf{v}}$ is the matrix representation of $S_{\text {rad }}$. We let $C$ denote the linear transformation sending $v_{i} \mapsto$ $\sum_{j=1}^{n} c_{j i} v_{j}$.

Then, letting $\mathbf{v}$ denote the column vector with $i$-th entry $v_{i}$,

$$
\begin{aligned}
\psi_{1}^{*}(d V)\left(v_{1}, \ldots, v_{n}\right) & =\operatorname{det}\left(U_{1}, d \psi_{1}\left(v_{1}\right), \ldots, d \psi_{1}\left(v_{n}\right)\right) \\
& =\operatorname{det}\left(U_{1}, \tilde{C}\left(v_{1}\right), \ldots, \tilde{C}\left(v_{n}\right)\right) \\
& =\operatorname{det}(C) \operatorname{det}\left(U_{1}, v_{1}, \ldots, v_{n}\right) \\
& =\rho \operatorname{det}(C) \operatorname{det}\left(\mathbf{n}, v_{1}, \ldots, v_{n}\right) \\
& =\operatorname{det}\left(I-r \cdot S_{\mathrm{rad}}\right) d M\left(v_{1}, \ldots, v_{n}\right),
\end{aligned}
$$

where $\mathbf{n}$ is the unit normal vector on $M_{i}$ in the same direction as $U$, and $\rho=\left\langle U_{1}, \mathbf{n}\right\rangle$. Hence, by (4.15) and the change of variables formula,

$$
\int_{\mathcal{B}_{i}} g d V=\int_{M_{i}} g \circ \psi_{1} \cdot \operatorname{det}\left(I-r S_{\text {rad }}\right) d M .
$$

This completes the proof.

\section{Medial version of the generalized Gauss-Bonnet theorem}

As a second consequence of Theorem 4.1, we deduce a form of the generalized Gauss-Bonnet theorem for the smooth boundary $\mathcal{B}$ of a generic region 
$\Omega \subset \mathbb{R}^{n+1}$ in terms of medial integrals. The standard generalized GaussBonnet formula applies in the case $n$ is even to give a formula for the Euler characteristic $\chi(\mathcal{B})$ in terms of an integral of the Gauss-Bonnet form over $\mathcal{B}$. In the case $n$ is odd $\chi(\mathcal{B})=0$, and there is no standard Gauss-Bonnet formula. There is Chern's version of the Gauss-Bonnet formula valid for an even-dimensional Riemannian manifold with boundary in terms of integrals on the manifold, which requires a form expressed in terms of a sum of characteristic forms [19, Vol. V]. We first give a single form of generalized Gauss-Bonnet which is valid for a region $\Omega \subset \mathbb{R}^{n+1}$ with compact closure and smooth boundary $\mathcal{B}$ independent of the dimension of $\mathcal{B}$.

Theorem 5.1 (generalized Gauss-Bonnet theorem). Suppose $\Omega \subset$ $\mathbb{R}^{n+1}$ is a region with compact closure and smooth boundary $\mathcal{B}$. Then,

$$
\frac{1}{s_{n}} \cdot \int_{\mathcal{B}} K_{n} d V=\chi(\Omega)
$$

and if $n$ is even

$$
=\frac{1}{2} \chi(\mathcal{B})
$$

Here $K_{n}$ is the Lipschitz-Killing curvature (which is the determinant of the differential geometric shape operator of $\mathcal{B}$ ) and $K_{n} d V$ is the GaussBonnet form. Also, $s_{n}=\operatorname{vol}\left(S^{n}\right)$ and $\chi(X)$ denotes the Euler characteristic of $X$.

Remark 5.2. In this version of Gauss-Bonnet, there is no restriction on how many connected components either $\mathcal{B}$ or $\Omega$ has. The outward pointing normal naturally provides a consistent orientation for each component.

This version of Gauss-Bonnet has the following medial version valid for all dimensions.

Theorem 5.3 (medial version of generalized Gauss-Bonnet theorem). Suppose $\Omega \subset \mathbb{R}^{n+1}$ is a region with compact closure and smooth generic boundary $\mathcal{B}$. Let $M$ be the Blum medial axis. Then,

$$
\frac{1}{s_{n}} \cdot \int_{\tilde{M}} K_{\mathrm{rad}} d M=\chi(\Omega)=\chi(M)
$$

and if $n$ is even

$$
=\frac{1}{2} \chi(\mathcal{B})
$$


with $s_{n}=\operatorname{vol}\left(S^{n}\right)$ and $\chi(X)$ as above.

Remark 5.4. Suppose instead that $(M, U)$ is a skeletal structure defining a region with smooth boundary $\mathcal{B}$ which satisfies the partial Blum condition. Then, the conclusion of Theorem 5.3 still applies.

Proof of Theorem 5.3. We first deduce Theorem 5.3 from Theorem 5.1. In the Gauss-Bonnet form $K_{n} d V, K_{n}=\operatorname{det}\left(S_{\mathcal{B}}\right)$ denotes the determinant of the differential geometric shape operator for $\mathcal{B}$ using the outward pointing unit normal vector field. Then, we apply the formula for $S_{\mathcal{B}}$ given by Theorem 4.1 of [7].

$$
S_{\mathcal{B}}=S_{\mathbf{v}}\left(I-r S_{\mathbf{v}}\right)^{-1}
$$

where $S_{\mathbf{v}}$ denotes the matrix representation of $S_{\text {rad }}$ with respect to a basis $\mathbf{v}$ for $T_{x} M$, as does $S_{\mathcal{B}}$ for a corresponding basis of $T_{x^{\prime}} \mathcal{B}$ with $x^{\prime}=\psi_{1}(x)$. Thus,

$$
K_{n}=\operatorname{det}\left(S_{\mathcal{B}}\right)=\frac{K_{\mathrm{rad}}}{\operatorname{det}\left(I-r S_{\mathbf{v}}\right)} .
$$

Using (4.1) to evaluate the integral in (5.1), and substituting in (5.3) yields the result for $\chi(\Omega)$. Then, the reverse of the radial flow provides a deformation retract of $\Omega$ onto $M$ by [7] (or see [20]). Thus, $\chi(\Omega)=\chi(M)$, completing the proof.

Proof of the Gauss-Bonnet theorem. We also provide the proof of the form of the Gauss-Bonnet theorem given here. First, as $\Omega$ is a smooth manifold with boundary $\mathcal{B}$, we may apply a standard formula from topology [21, Section 28]

$$
\chi(2 \Omega)=2 \cdot \chi(\Omega)-\chi(\mathcal{B})
$$

where $2 \Omega$ denotes the double of $\Omega$ obtained by attaching two copies of $\Omega$ along $\mathcal{B}$. If $n$ is odd $\chi(\mathcal{B})=0$; however, if $n$ is even, $2 \Omega$ is odd-dimensional and without boundary so $\chi(2 \Omega)=0$, and by $(5.4), \chi(\mathcal{B})=2 \cdot \chi(\Omega)$. This yields the second part of Theorem 5.1 from the first part. Thus, we need to show

$$
\chi(\Omega)=\operatorname{deg}(\text { Gauss map of } \mathcal{B}) .
$$

For the first part, we follow the standard argument with one change. The degree of the Gauss map on $\mathcal{B}$ using the outward pointing normal is computed by $1 / s_{n} \cdot \int_{\mathcal{B}} K_{n}$. We compute this a second way using Morse theory on $\Omega$. 
We may flow in along the inward pointing unit normal vector field on $\mathcal{B}$ to define a collar neighborhood of $\mathcal{B}$ in $\Omega$. If $t$ denotes the distance along the flow lines, then $t$ is a smooth function on the collar neighborhood which is 0 on $\mathcal{B}$ and has no critical points. We may extend $t$ to a smooth function on $\Omega$. We may perturb this function to a Morse function $f$ on $\Omega$ which agrees with $t$ near $\mathcal{B}$ and has $f^{-1}(0)=\mathcal{B}$. Let $\left\{x_{1}, \ldots, x_{k}\right\}$ denote the critical points of $f$ with $\lambda_{i}$ the Morse index of $x_{i}$. Then, by Morse theory

$$
\chi(\Omega)=\chi(\mathcal{B})+\sum_{i=1}^{k}(-1)^{\lambda_{i}} .
$$

Second, $\operatorname{ind}_{x_{i}}(-\nabla f)$, the index of the negative gradient vector field $-\nabla f$ at $x_{i}$ is given by

$$
\operatorname{ind}_{x_{i}}(-\nabla f)=(-1)^{n+1} \cdot \operatorname{ind}_{x_{i}}(\nabla f)=(-1)^{n+1} \cdot(-1)^{\lambda_{i}} .
$$

Also, choose around each $x_{i}$ small disjoint open balls $B_{i}$ which also are disjoint from $\mathcal{B}$. We let $S_{i}$ denote the boundary sphere of $B_{i}$. Then, $G(x)=$ $-\nabla f(x) /\|\nabla f(x)\|$ is a smooth map on $\Omega \backslash \cup_{i=1}^{k} B_{i} \rightarrow S^{n}$ which agrees with the Gauss map on $\mathcal{B}$. Hence,

$$
\operatorname{deg}(G \mid \mathcal{B})=\sum_{i=1}^{k} \operatorname{deg}\left(G \mid S_{i}\right) .
$$

However,

$$
\operatorname{deg}\left(G \mid S_{i}\right)=\operatorname{ind}_{x_{i}}(-\nabla f)=(-1)^{n+1} \cdot(-1)^{\lambda_{i}} .
$$

Hence, using (5.6) and (5.7), (5.5) becomes

$$
\chi(\Omega)=\chi(\mathcal{B})+(-1)^{n+1} \cdot \operatorname{deg}(G \mid \mathcal{B}) .
$$

Finally, if $n$ is odd, $\chi(\mathcal{B})=0$ so (5.8) gives the result; while if $n$ is even, $\chi(\mathcal{B})=2 \cdot \chi(\Omega)$, and again (5.8) gives the result.

\section{Integrals over regions as skeletal integrals}

Throughout this section, we consider a skeletal structure $(M, U)$ which defines a region $\Omega$ with possibly singular boundary except that we do not assume the partial Blum condition is satisfied. This is the "non-Blum case", 
but we still show that we still can represent integrals over a region $\Omega$ as skeletal integrals. Of course the result is still valid in the partial Blum case. We suppose that $g: \Omega \rightarrow \mathbb{R}$ is a Borel measurable and integrable function (with respect to Lebesgue measure). We let $\tilde{\psi}$ denote the radial flow from $\tilde{M}$, and let $g_{1}=g \circ \tilde{\psi}$ denote the function on the "positive normal bundle" on $\tilde{M}$, see Section 2 and [7, Section 4]. Let $g_{1}(x, t)=g(x+t U(x))$. Provided the integral is defined, we let

$$
\tilde{g}(x)=\int_{0}^{1} g_{1}(x, t) \cdot \operatorname{det}\left(I-\operatorname{tr} S_{\mathrm{rad}}\right) d t
$$

Theorem 6.1. Suppose $(M, U)$ is a skeletal structure which defines a region $\Omega$ with possibly singular boundary $\mathcal{B}$ (without being partially Blum). Let $g: \Omega \rightarrow \mathbb{R}$ be Borel measurable and integrable (for Lebesgue measure). Then, $\tilde{g}$ is defined for almost all $x \in \tilde{M}$, it is integrable on $\tilde{M}$, and

$$
\int_{\Omega} g d V=\int_{\tilde{M}} \tilde{g} \cdot r d M .
$$

Remark 6.2. By a change of variables $t^{\prime}=r \cdot t$, we may instead write the formula (6.2) as the integral of $\tilde{g}^{\prime}$, where now

$$
\tilde{g}^{\prime}=\int_{0}^{r} g\left(x+t^{\prime} U_{1}(x)\right) \cdot \operatorname{det}\left(I-t^{\prime} S_{\mathrm{rad}}\right) d t .
$$

Before proving this theorem, we deduce several immediate consequences. First, we deduce a "Crofton-type formula" for integrals over regions $\Gamma \subset \Omega$. Such a formula computes integrals over the regions by first integrating over the intersection of the region with radial lines (figure 6), and then integrating the resulting function over the skeletal set $M$ which parametrizes such lines.

We let

$$
\tilde{g}_{\Gamma}(x)=\int_{0}^{1} \chi_{\Gamma} \cdot g_{1}(x, t) \cdot \operatorname{det}\left(I-\operatorname{tr} S_{\mathrm{rad}}\right) d t,
$$

where $\chi_{\Gamma}$ is the characteristic function of $\Gamma$.

Corollary 6.3 (medial Crofton-type formula). Suppose $(M, U)$ is a skeletal structure which defines a region $\Omega$ with possibly singular boundary $\mathcal{B}$ (without being partially Blum). Let $\Gamma \subset \Omega$ be Borel measurable and let $g: \Gamma \rightarrow \mathbb{R}$ be Borel measurable and integrable for Lebesgue measure. Then, 


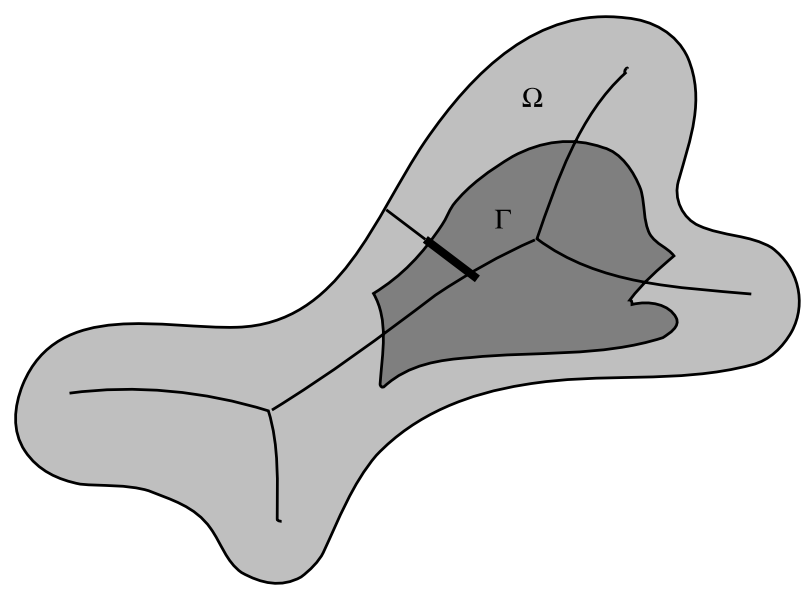

Figure 6: Integration over regions $\Gamma \subset \Omega$ as skeletal integrals.

$\tilde{g}$ is defined for almost all $x \in \tilde{M}$; it is integrable on $\tilde{M}$ and

$$
\int_{\Gamma} g d V=\int_{\tilde{M}} \tilde{g}_{\Gamma} \cdot r d M .
$$

Note that $\tilde{g}_{\Gamma}$ will vanish for all $(x, U(x))$ for which the radial line $\{x+$ $t U(x): 0 \leq t \leq 1\}$ only intersects $\Gamma$ in a set of measure 0 .

Proof of Corollary 6.3. We apply Theorem 6.1 to the function $\chi_{\Gamma} \cdot g$ just as in the proof of Corollary 4.2, we applied Theorem 4.1 to $\chi_{R} \cdot g$.

Second, we use Theorem 6.1 for computing the volume of $\Omega$. Let

$$
\delta(x)=\int_{0}^{1} \operatorname{det}\left(I-\operatorname{tr} S_{\mathrm{rad}}\right) d t .
$$

Then, we can compute the volume of $\Omega$ in terms of an integral of $\delta$ over $\tilde{M}$.

Theorem 6.4. Suppose $(M, U)$ is a skeletal structure which defines a region $\Omega$ with possibly singular boundary $\mathcal{B}$ (without being partially Blum). Then

$$
\text { Volume of } \Omega=\int_{\tilde{M}} \delta \cdot r d M
$$

Proof of Theorem 6.4. We just apply Theorem 6.1 in the case $g \equiv 1$. 


\subsection{Weyl expansion of integrals for general regions}

We expand the integrand in the RHS of (6.2) to understand its relation with Weyl's formula. First, we use (4.5) to expand (6.1).

$$
\delta(x)=\sum_{i=0}^{n}(-1)^{i} m_{i}(g) \sigma_{r i} \cdot r^{i},
$$

where

$$
m_{i}(g)(x)=\int_{0}^{1} g(x+t U(x)) \cdot t^{i} d t
$$

is an $i$-th radial moment of $g$ along the radial line $\{x+t U(x): 0 \leq t \leq 1\}$. Then, we can expand (6.2) as a sum of weighted integrals of radial moments;

$$
\int_{\Omega} g d V=\sum_{i=0}^{n}(-1)^{i} I_{i+1}\left(m_{i}(g) \cdot \sigma_{r i}\right) .
$$

In the special case when $g \equiv 1$, we obtain an expansion of the formula for the volume of $\Omega$.

$$
\text { Volume of } \Omega=\sum_{i=0}^{n} \frac{(-1)^{i}}{i+1} I_{i+1}\left(\sigma_{r i}\right) \text {. }
$$

Example 6.5. Suppose $\Omega \subset \mathbb{R}^{2}$ or $\mathbb{R}^{3}$ has compact closure with smooth generic boundary so the Blum medial axis $M$ together with the radial vector field $U$ defines a skeletal structure $(M, U)$.

In the first case,

$$
\delta(x)=\int_{0}^{1} 1-t r \kappa_{r} d t=1-\frac{1}{2} r \kappa_{r} .
$$

Hence, we may compute the area of a 2-dimensional region $\Omega$

$$
\operatorname{area}(\Omega)=\int_{\tilde{M}} r d M-\frac{1}{2} \int_{\tilde{M}} r^{2} \kappa_{r} d M .
$$

For a 3-dimensional region $\Omega$,

$$
\delta(x)=1-\frac{1}{2} r H_{\mathrm{rad}}+\frac{1}{3} r^{2} K_{\mathrm{rad}}
$$


Thus,

(6.11) volume $(\Omega)=\int_{\tilde{M}} r d M-\frac{1}{2} \int_{\tilde{M}} r^{2} H_{\mathrm{rad}} d M+\frac{1}{3} \int_{\tilde{M}} r^{3} K_{\mathrm{rad}} d M$.

Remark 6.6. In Section 7, we obtain analogs of (6.8) and (6.9) where we replace $\Omega$ by a region $\Gamma$ which is a union of radial lines over a region $\tilde{R}$ in $\tilde{M}$. This includes the case when $M$ is a compact smooth manifold without boundary, so we obtain integral formulas for volumes of various generalized and partial tubes and offset regions.

Proof of Theorem 6.1. First, we consider the definition of $\tilde{g}(x)$ by the integral in Equation (6.1). It is enough to consider a neighborhood $W$ of a point $x_{0}$ and show that $\tilde{g}(x)$ is defined for almost all $x \in W$ and integrable on $W$. For then as $\tilde{M}$ is compact, we can cover $\tilde{M}$ by a finite number of such neighborhoods so $\tilde{g}(x)$ is defined a.e. and integrable on $\tilde{M}$. Now any point has a paved neighborhood which is a finite union of compact manifolds with boundaries and corners. Thus, it is sufficient to establish a formula for the restriction to a single compact manifold $M_{i}$ with boundaries and corners whose interior consists of regular points of $M$ (with a single smooth value of $U$ defined on $M_{i}$ ), the positive normal bundle has the form $M_{i} \times[0, \infty)$, and $\tilde{\psi}$ is given by $\tilde{\psi}(x, t)=x+t U(x)$. The differentiable map $\tilde{\psi}: M_{i} \times[0,1] \rightarrow \mathbb{R}^{n+1}$ is a diffeomorphism for $0<t<1$ and hence is one-one except on the boundary. Its image is compact and hence a Borel set, on which $g$ is integrable. Thus, $g_{1}$ is integrable so we may apply Fubini's Theorem to conclude that for almost all $x \in M_{i}$, the integral in (5.1) is defined and the resulting function defined a.e. on $M_{i}$ is integrable. Hence, $\tilde{g}(x)$ is defined a.e. and integrable on $\tilde{M}$.

Now, we proceed with a derivation of the formula. The proof will be similar to that for Theorem 4.1 .

By a partition of unity argument using paved neighborhoods, we may reduce to establishing the formula again for the case of a single compact manifold $M_{i}$ with boundaries and corners whose interior consists of regular points of $M$ (with a single smooth value of $U$ defined on $M_{i}$ ). Then, $\psi: M_{i} \times$ $[0,1] \rightarrow \mathbb{R}^{n+1}$ is a diffeomorphism on the interior. Hence, if $\Omega_{i}=\psi\left(M_{i} \times\right.$ $[0,1])$, then it is sufficient to show

$$
\int_{\Omega_{i}} g d V=\int_{\tilde{M}_{i}} \tilde{g} \cdot r d M
$$


For this, we again use the change of variables formula for $\psi$. We let $\left\{v_{1}, \ldots\right.$, $\left.v_{n}\right\}$ be a basis of $T_{x} M_{i}$. Since $\frac{\partial \psi}{\partial t}=U$, we compute

$$
\psi^{*} d V\left(\frac{\partial}{\partial t}, v_{1}, \ldots, v_{n}\right)=\operatorname{det}\left(U, d \psi\left(v_{1}\right), \ldots, d \psi\left(v_{n}\right)\right) .
$$

Using (2.1) and proceeding as in (4.14), except letting $\tilde{C}=\left(I-t r \cdot S_{\mathbf{v}}\right)^{\mathrm{T}}$, we obtain

$$
\begin{aligned}
\psi^{*} d V\left(\frac{\partial}{\partial t}, v_{1}, \ldots, v_{n}\right) & =r \cdot \operatorname{det}\left(U_{1}, \tilde{C}\left(v_{1}\right), \ldots, \tilde{C}\left(v_{n}\right)\right) \\
& =r \cdot \rho \operatorname{det}(C) \operatorname{det}\left(\mathbf{n}, v_{1}, \ldots, v_{n}\right) \\
& =r \cdot \operatorname{det}\left(I-\operatorname{tr} S_{r a d}\right) d M\left(v_{1}, \ldots, v_{n}\right) .
\end{aligned}
$$

Hence, by the change of variables formula and Fubini's theorem,

$$
\int_{\Omega_{i}} g d V=\int_{M_{i}} \int_{[0,1]} g \circ \psi \cdot r \cdot \operatorname{det}\left(I-\operatorname{tr} S_{\mathrm{rad}}\right) d M,
$$

and carrying out the innermost integral,

$$
=\int_{M_{i}} \tilde{g} \cdot r d M
$$

where

$$
\tilde{g}=\int_{[0,1]} g \circ \psi \cdot \operatorname{det}\left(I-\operatorname{tr} S_{\mathrm{rad}}\right) d t
$$

Hence

$$
\int_{\Omega_{i}} g d V=\int_{M_{i}} \tilde{g} \cdot r d M
$$

as claimed.

\section{Volumes of generalized tubes}

In [11], Gray gives an encyclopedic treatment of volumes of tubes on manifolds in numerous settings. We consider here the generalizations of tubes by allowing skeletal structures, or considering tubes on smooth manifolds without requiring normal directions nor constant radii values, and allowing partial tubes over subregions. We generally refer to such tubes as generalized or partial tubes. To determine formulas for the volumes of such tubes, we 
return to consequences of Theorem 6.4 and Corollary 6.3 using the expansion (6.9).

We consider a skeletal structure $(M, U)$ defining a region with either a smooth or possibly singular boundary $\mathcal{B}$. As already mentioned, the general results we obtain already suggest that a region $\Omega$ with smooth generic boundary $\mathcal{B}$ and Blum medial axis $M$ is a "generalized tube on $M$ ". As we specialize the conditions on both $M$ and $U$, the regions begin to resemble our usual notions of tubes.

\subsection{Types of generalized or partial tubes}

(1) Generalized or partial tubes on a skeletal set $M$. For a skeletal structure $(M, U)$ (defining a region with smooth or possibly singular boundary $\mathcal{B}$ ), we may suppose $r$ is constant (but $U$ need not be normal, nor need $M$ be a smooth manifold without boundary) and obtain a generalized tube on $M$ (figure $7 \mathrm{a}$ ); or we may restrict the tube to a region in $\tilde{M}$ (figure $7 \mathrm{~b}$ ).

(2) Generalized or partial or half-tube on a smooth manifold. Alternately, we may suppose $M$ is a compact smooth $n$-dimensional submanifold without boundary of $\mathbb{R}^{n+1}$, with a smooth multivalued vector field $U$ so that $(M, U)$ is a skeletal structure (defining a region with smooth or possibly singular boundary $\mathcal{B}$ ). First, we consider the generalized tube where $r$ is not constant nor need $U$ be normal to $M$. We then further specialize to cases where either $U$ is normal, or $r$ is constant (with the same value for both sides of $M$ ), and finally to where both hold and we return to a traditional tube (as in Weyl's formula).

We examine the form that volume formulas take for these cases, including the special forms for the formulas as a result of special conditions. These

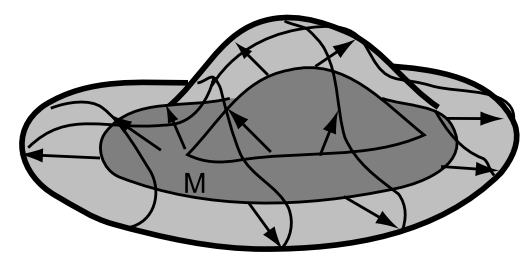

a)

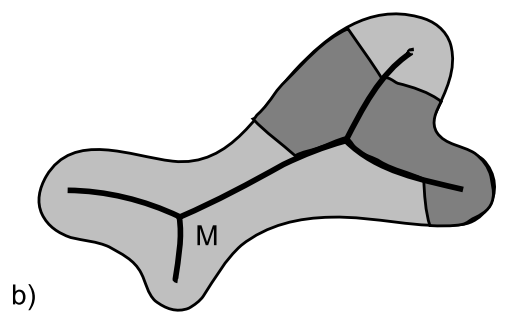

Figure 7: Generalized and partial tubes on a skeletal set $M$. 
integrals will involve global invariants

$$
K_{r i}=\int_{\tilde{M}} \sigma_{r i} d M
$$

where as earlier $\sigma_{r i}$ denotes the $i$-th elementary symmetric function in the principal radial curvatures (some of which may be complex in the non-Blum case, although $\sigma_{r i}$ will be real-valued). As well, we consider for a measurable region $\tilde{R}$ in $\tilde{M}$ and the partial tubes on $\tilde{R}$. In this case, we shall consider instead the global invariants on $\tilde{R}$ defined by integrals

$$
K_{r i}(\tilde{R})=\int_{\tilde{R}} \sigma_{r i} d M .
$$

\subsection{Generalized or partial tube on a skeletal set}

In the first case for a skeletal set $M$, with $r$ constant but $U$ non-normal, we can directly apply (6.9) to obtain a Weyl-type expansion for volume which is a polynomial in $r$.

Corollary 7.1. For a generalized constant radius tube $\Omega$ on a skeletal set $M$,

$$
\text { Volume of } \Omega=\sum_{i=0}^{n} \frac{(-1)^{i}}{i+1} K_{r i} \cdot r^{i+1} \text {. }
$$

7.2.1. Partial tube on a skeletal set. Second, we may consider a constant radius tube on only part of $M$ as in figure $7 \mathrm{~b}$. Let $\tilde{R}$ denote a measurable region in $\tilde{M}$. We let $\Gamma$ denote the union of the radial lines from points of $\tilde{R}$. Equivalently, $\Gamma=\psi\left(N_{1} \mid \tilde{R}\right)$, where $N_{1}$ denotes the subset of the positive normal bundle of vectors of length $\leq 1$. We can decompose $\tilde{R}$ into a finite union of measurable sets $R_{i}$ contained in compact manifolds with boundaries and corners $\tilde{M}_{i}$, which only intersect in sets of measure 0 , and on which the values of $U$ define a smooth vector field. Then, $\Gamma$ is a union of the measurable sets $\Gamma_{i}=\psi\left(R_{i} \times[0,1]\right)$ which only intersect on sets of measure 0 . Thus, $\Gamma$ is a measurable region (whose volume is the sum of the volumes of the $\Gamma_{i}$ ). In turn, we may apply Corollary 6.3 with $g \equiv 1$ to each $\Gamma_{i}$ and sum over $i$ to obtain

Corollary 7.2. For a constant radius partial tube $\Gamma$ on a region $\tilde{R}$,

$$
\text { Volume of } \Gamma=\sum_{i=0}^{n} \frac{(-1)^{i}}{i+1} K_{r i}(\tilde{R}) \cdot r^{i+1} \text {. }
$$




\subsection{Generalized and partial tubes on smooth manifolds}

Next, we turn to the second class of generalized tubes where $M$ is a compact smooth $n$-dimensional submanifold of $\mathbb{R}^{n+1}$ (without boundary). Then, for a skeletal structure $(M, U), U$ is defined by a pair of smooth non-vanishing vector fields which at each point $x \in M$, point to opposite sides of $M$. Also, $\tilde{M}$ consists of two copies of $M$, one corresponding to each side of $M$.

If we place no restriction on $r$ or $U$, then we still obtain the same formulas (6.9) and the analogue for regions using the expansion for (6.4). When we further restrict $r$ or $U$, we do obtain more specialized forms which yield analogs of Weyl's formula and other classical formulas.

7.3.1. Constant radius generalized tube on a smooth manifold. In the case that $r$ is constant without $U$ necessarily being normal on $M$, we obtain a polynomial expansion for the volume given by (7.3).

7.3.2. Varying radius tube on a smooth manifold. Next, we consider instead the case where $U$ is normal to $M$, but $r$ varies (except that all of its values at a point agree).

Corollary 7.3 (generalized Weyl's formula for a varying radius tube). For a tube $\Omega$ defined on a smooth manifold $M$, but with varying radius $r$,

$$
\text { Volume of varying radius tube } \Omega=2 \cdot \sum_{i=0}^{[n / 2]} \int_{M} \sigma_{2 i} \cdot r^{2 i+1} d V \text {. }
$$

Here $\sigma_{2 i}$ denotes the $2 i$-th elementary symmetric function in the principal curvatures of $M$ (which is independent of the orientation).

In the special case of a true tube, with $r$ constant and $U$ normal, we may take out $r$ from the integrals and obtain Weyl's tube formula for this case.

Proof. As $U$ is normal to $M, S_{\text {rad }}$ is the usual differential geometric shape operator for each side of $M$, using the normal vector field pointing in that direction. If we denote these by $S_{\text {rad }}^{(i)}, i=1,2$, then the principal curvatures on each side differ by a sign, so as in the proof of Corollary $4.5, \sigma_{r i}^{(1)}=$ $(-1)^{i} \sigma_{r i}^{(2)}$. Thus, in (6.9), the integrals on each side of $M$ will cancel for $i$ odd, and will be equal for $i$ even, yielding the desired formula. 
7.3.3. Partial and half-tubes on a smooth manifold. A partial tube $\Gamma$ on a smooth manifold is defined by two measurable regions $R_{i}, i=1,2$ on $M$, each one associated to a side of $M$. Then, $\Gamma$ is the union of the radial lines from these points on each side, all of length $r$. The volume of this partial tube is the sum of the volumes of the partial tubes on each side. Hence, it is enough to compute the volume of a partial tube for a one-sided region $R$. We let $U$ denote the smooth vector field on the side corresponding to $R$. Then, we may naturally identify $R$ with the copy of $R$ in $\tilde{M}$ corresponding to the side associated to $U$. We can apply the earlier formulas obtained for a general skeletal set but now with $\tilde{R}=R$. In the case when neither $r$ is constant nor $U$ is normal, then we only obtain the general formula (6.9). If the radius $r$ is constant, without $U$ being normal, then we obtain the volume of constant radius partial tube for $\tilde{R}=R$ given by (7.4). In the case that $R=M$ is a one-sided region, we obtain a "half-tube". Then, (6.9) takes the following simplified form.

Corollary 7.4. The volume of a varying radius half-tube $\Gamma$ is given by

$$
\text { Volume of } \Gamma=\sum_{i=0}^{n} \frac{(-1)^{i}}{i+1} \int_{M} \sigma_{r i} \cdot r^{i+1} d V \text {. }
$$

Remark 7.5. We make two remarks regarding volumes of half-tubes. The first is that if we are only given one vector field $U^{(1)}$ pointing toward one side of $M$, for which the radial flow satisfies the conditions for a region with possibly singular boundary, then we may choose a normal vector field $U^{(2)}$ of sufficiently small constant length and pointing to the opposite side from $U^{(1)}$. Together, the $U^{(i)}$ define a multivalued vector field $U$ on $M$, so that $(M, U)$ is a skeletal structure which defines a region with possibly singular boundary. Then the preceding formula (7.6) applies to the half-tube determined by $U^{(1)}$.

Second, (7.6) remains valid even in the case that the boundary of the region defined by $(M, U)$ is singular in the sense of Definition 2.1.

\subsection{Generalized offset regions and a "mining rights formula"}

We consider three consequences of the preceding remarks for generalized offset regions, for "signed offset regions" and a "mining rights formula" proposed by Stetten [22] which gives an alternate way to compute the volume of a region. 
7.4.1. Volumes of offset regions and Steiner's formula. Suppose that $\Omega$ is a compact region in $\mathbb{R}^{n+1}$ with a smooth boundary $\mathcal{B}$. Let $U$ be a smooth nonvanishing outward pointing vector field. We construct a generalized offset region. Suppose $(\mathcal{B}, U)$ satisfies the conditions for defining a region $\Omega^{\prime}$ with possibly singular boundary. We refer to $\Omega^{\prime}$ as a generalized offset region (with possibly singular boundary).

In the standard case of an offset region using a normal vector field of constant length, there is a generalization of the classical Steiner's formula for regions with smooth boundary [11, Chapter 10]. In fact, the offset region is a half-tube. The generalized offset region is a generalized half-tube; hence by Remark 7.5 and (7.6), we obtain the following formula.

Corollary 7.6. The volume of the generalized offset region $\Omega^{\prime}$ is given by

$$
\text { Volume of generalized offset region }=\sum_{i=0}^{n} \frac{(-1)^{i}}{i+1} \int_{M} \sigma_{r i} \cdot r^{i+1} d M \text {. }
$$

Remark 7.7. We emphasize that $d M$ denotes the medial measure (even though it is being used on $M$ ) and it automatically takes into account the failure of the vector field $U$ to be non-normal.

In the special case that the offset region has constant radius, we may remove $r$ from the integrals in (7.7) and obtain a polynomial expansion in $r$. If the offset region is defined using a normal vector field, then $\sigma_{r i}$ is the $i$-th elementary symmetric function in the principal curvatures of $M$.

7.4.2. Integrals and volumes for "signed offset regions". Suppose $U_{1}$ is a smooth unit vector field on $M$, nowhere tangent to $M$. We let $r$ be a smooth function on $M$ which may be positive and negative. We let $U=r \cdot U_{1}$. If we try to define a generalized offset region using $U$, then we have a basic problem: depending on the sign of $r, U$ points to different sides of $M$. Nonetheless, we suppose for simplicity that $r^{-1}(0)$ is a piecewise smooth $n-1$ submanifold separating the regions $R_{+}$and $R_{-}$where $r$ is positive and negative. Suppose $r$ also satisfies

$$
|r|<\min \frac{1}{\left|\kappa_{r i}\right|} \text { for all } \kappa_{r i} \text { such that } \operatorname{sgn}(r) \cdot \kappa_{r i}>0 .
$$

Then the radial flow does not develop singularities from $R_{+}$nor from $R_{-}$. We suppose that the flow remains globally one-one on each of these regions. We denote the image of the radial flow at time one by $\mathcal{B}$ and the region 


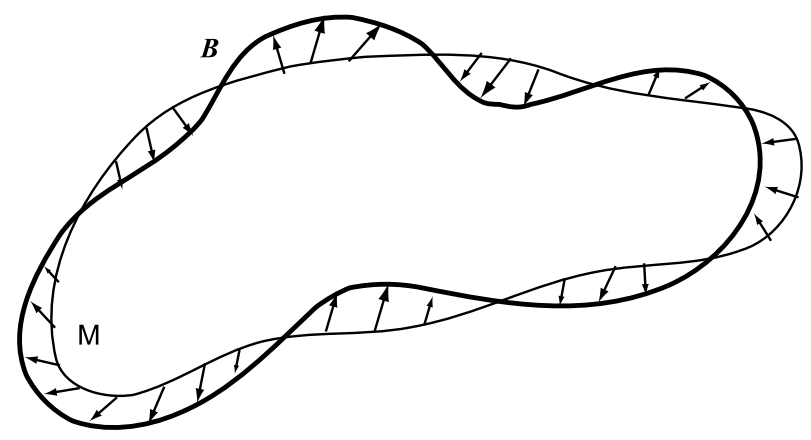

Figure 8: Signed offset region $\Omega$ on $M$.

between $M$ and $\mathcal{B}$ by $\Omega$. We refer to this region as a signed offset region (figure 8).

Then, $\Omega$ is made up of partial tubes $\Omega_{+}$and $\Omega_{-}$on the regions $R_{+}$ and $R_{-}$on each side of $M$ (actually $\mathcal{B}$ and $M$ intersect on the boundaries; however, the arguments we have given will still apply). We suppose we are given a non-negative function $g$ on $\Omega$, and we want to integrate $g$ on $\Omega$, except we want to treat the values of $g$ over $\Omega_{-}$as being negative. This is represented by the following signed offset integral

$$
\int_{\Omega} \operatorname{sgn}(r) \cdot g d V
$$

We apply the preceding results regarding partial tubes (except to integrals rather than just volumes) to $\Omega_{+}$and $\Omega_{-}$separately. We define for $\varepsilon= \pm 1$

$$
\delta_{\varepsilon}(x)=\int_{0}^{1} g(x+t \varepsilon U(x)) \cdot \operatorname{det}\left(I-t r S_{\mathrm{rad}}\right) d t .
$$

Corollary 7.8. For the signed offset region $\Omega$, and non-negative integrable function $g$,

$$
\int_{\Omega} \operatorname{sgn}(r) \cdot g d V=\int_{R_{+}} r \cdot \delta_{+} d M-\int_{R_{-}} r \cdot \delta_{-} d M .
$$

In the case that $g \equiv 1$, we obtain from (7.9) the signed volume of $\Omega$, which measures the difference in volume between the region enclosed by $\mathcal{B}$ and that enclosed by $M$. This is a difference of volumes of partial tubes, and is given by the preceding formulas as a difference of integrals.

7.4.3. "Mining rights formula" for volume of a region. If $\Omega$ is a compact region with generic smooth boundary $\mathcal{B}$ in $\mathbb{R}^{n+1}$, then an alternate 
approach to finding the volume of $\Omega$ is to express it as an integral over $\mathcal{B}$. Define the function $\delta^{\prime}$ by the expression

$$
\delta^{\prime}=\int_{0}^{r} \prod\left(1-t \kappa_{i}\right) d t
$$

where the $\kappa_{i}$ are the principal curvatures of $M$ (with respect to the inward pointing normal). Then, the following formula (7.10) was proposed by Stetten [22] who called it the "mining rights formula".

Theorem 7.9. If $\Omega$ is a region in $\mathbb{R}^{n+1}$ with generic smooth boundary $\mathcal{B}$, then the volume of $\Omega$ is given by the following formula

$$
\text { Volume of } \Omega=\int_{\mathcal{B}} \delta^{\prime} d V \text {. }
$$

We derive this formula as a consequence of the formula obtained earlier for the volume of a half-tube allowing a singular boundary.

Proof. We define on $\mathcal{B}$ the vector field $U$ which at a point $x$ is from $x$ to the image in the Blum medial axis under the grassfire flow. In the generic case this is a continuous piecewise smooth vector field (it is the negative of the translate of the radial vector field from the medial axis $M$ to $\mathcal{B}$. Then, we invert our view of $\Omega$ as being built from the medial axis $M$ by the radial flow. Instead, it is the half-tube on $\mathcal{B}$ defined by this vector field. Thus, we flow inward from $\mathcal{B}$ by the grassfire flow to obtain the half-tube $\Omega$ with "singular boundary" $M$ as in figure 9.

In order to apply half-tube formula in this case, we have to break up $M$ into regions on which $U$ is smooth and use the formula for partial tubes on the regions. Then adding up these formulas, we still obtain the formula (7.6)

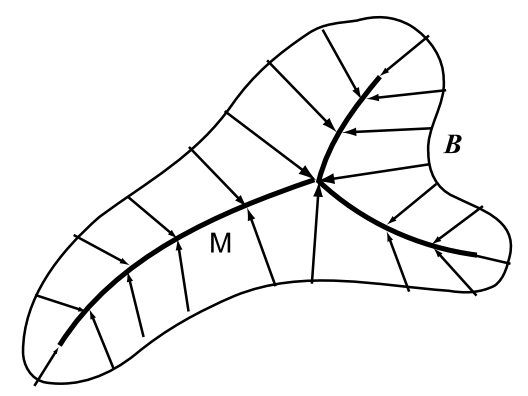

Figure 9: Region $\Omega$ as a half-tube on $\mathcal{B}$ with singular boundary $M$. 
for the volume of $\Omega$. We first rewrite this as an integral from Theorem 6.4.

$$
\text { Volume of } \Omega=\int_{\mathcal{B}} r \cdot \delta(x) d V
$$

where

$$
\begin{aligned}
r \cdot \delta(x) & =\int_{0}^{1} \operatorname{det}\left(I-t \cdot r \cdot S_{\mathcal{B}}\right) \cdot r \cdot d t \\
& =\int_{0}^{1} \prod\left(I-t \cdot r \cdot \kappa_{i}\right) \cdot r \cdot d t .
\end{aligned}
$$

Here the $\kappa_{i}$ are the principal curvatures of $\mathcal{B}$ but for the inward pointing normal. Then, after performing a change of coordinates $s=r \cdot t$, we see that $r \cdot \delta=\delta^{\prime}$; hence, (7.11) yields the formula.

\section{Divergence theorem for fluxes with discontinuities across the medial axis}

Let $(M, U)$ be a skeletal structure which defines $\Omega$ as a region with smooth boundary $\mathcal{B}$ in $\mathbb{R}^{n+1}$. For example, $M$ could be the Blum medial axis $M$ of $\Omega$ in the case of a smooth generic boundary $\mathcal{B}$. We will derive a version of the divergence theorem for a region $\Gamma \subset \Omega$ for a vector field $F$ which may have discontinuities across $M$. Before we begin defining exactly what we will mean, we first introduce a piece of terminology. Let $M_{\alpha}$ denote either a component of a stratum of $M$ or a manifold with boundary or corners appearing in a paved neighborhood in $M$. We let $\tilde{M}_{\alpha}$ denote the submanifold in $\tilde{M}$ given by $\tilde{M}_{\alpha}$ together with a choice of smooth value of $U$ on $M_{\alpha}$. Then, for the radial flow $\psi$, we will refer to the image $\psi\left(\tilde{M}_{\alpha} \times[0,1]\right)$ as the radial trace of $\tilde{M}_{\alpha}$. By our assumptions on $(M, U)$ (see Section 2 and [7]), it will still be a smooth manifold if $M_{\alpha}$ is a stratum, or a manifold with boundary and corners if $M_{\alpha}$ is one. We denote the radial trace more simply as $\tilde{M}_{\alpha \psi}$. In figure 10 , we see the radial traces of the singular stratum denoted by heavier lines, with the darker region denoting the radial traces of the submanifolds $\tilde{M}_{i j}$.

Definition 8.1. A vector field $F$ will be a smooth vector field on $\Omega$ with (possible) discontinuities across $M$, if $F$ is a smooth vector field on $\Omega \backslash M$ which in addition has the following property at any point $x_{0} \in M$. There is a neighborhood $V$ of $x_{0}$ in $\mathbb{R}^{n+1}$ and a paved neighborhood $W \subset V$ of $x_{0}$ with $W=\cup M_{i}$ a decomposition into manifolds with boundaries and corners 


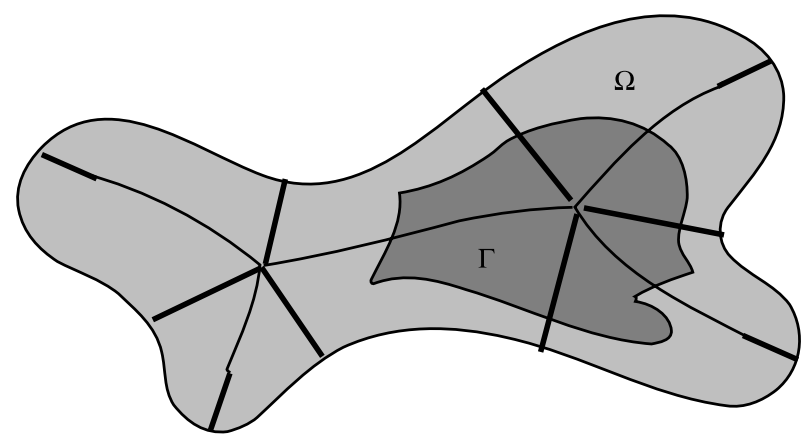

Figure 10: Radial traces and a region $\Gamma$ in $\Omega$ in radial general position.

so that: if $\tilde{M}_{i}$ denotes $M_{i}$ with a chosen smooth value of $U$, and $\tilde{M}_{i}$ belongs to the abstract neighborhood of $C$, a local complementary component of $M$ at $x_{0}$ in $V$, then $F$ extends smoothly to the radial trace $\tilde{M}_{i \psi}$.

Example 8.2. We may translate $U$ along each radial line to obtain a vector field (again denoted by $U$ ) on $\Omega$, which is multivalued on $M$ but smooth on $\Omega \backslash M$. Thus, this $U$ is smooth with discontinuities across $M$. Likewise, the corresponding unit vector field $U_{1}$ analogously obtained by translation is also smooth with discontinuities across $M$. In the Blum case, $-U_{1}$ is the vector field corresponding to the "grassfire flow" (i.e., eikonal flow) considered by Siddiqi et al. in $[13,15]$.

As a consequence of Definition 8.1, $F$ extends to a multivalued continuous piecewise smooth vector field on $M$. Also, div $F$ also extends smoothly to each radial trace $\tilde{M}_{i \psi}$.

Next, we specify the types of regions $\Gamma$ over which we define integrals of $\operatorname{div} F$, as well as flux integrals of $F$ over the boundary $\partial \Gamma$, with the goal of finding the appropriate generalization of the divergence theorem relating them.

Normally, flux integrals are defined over smooth boundaries. However, as in [23], we can define the flux integral of a vector field over a manifold with boundaries and corners and there is still a version of the divergence theorem [23, Theorem 7.1]. Now we consider a region $\Gamma \subset \Omega$ which has regular piecewise smooth boundary $\partial \Gamma$. By this, we shall mean that each point $x_{0}$ of $\partial \Gamma$ has a paved neighborhood $V$ in $\Gamma$. Furthermore, we require that $\Gamma$ is in radial general position, which means that $M$ and the radial traces of $M_{\text {sing }}$ decompose $\Gamma$ into a union of regions with regular piecewise smooth boundary. 
For example, if $\Gamma$ has a regular piecewise smooth boundary and the various dimensional smooth pieces of $\partial \Gamma$ are transverse to $M$ and the radial traces of its strata as in figure 10, then $\Gamma$ is in radial general position.

Since $\operatorname{div} F$ is bounded and smooth on $\Omega \backslash M$, and $M$ is a set of measure 0 in $\Omega$, we may extend $\operatorname{div} F$ any way we wish to $M$ to obtain a measurable function. As $\Gamma$ is a Borel set and compact, the integral $\int_{\Gamma} \operatorname{div} F d V$ is defined. Likewise, as $\partial \Gamma$ can be locally paved by compact manifolds with boundaries and corners with outward pointing normal vectors $\mathbf{n}_{\Gamma}$, the integral $\int_{\partial \Gamma} F \cdot \mathbf{n}_{\Gamma} d S$ is defined, where $d S$ denotes the $n$-dimensional volume over the faces of $\partial \Gamma$. To give a version of the divergence theorem, we define a multivalued function $c_{F}$ on $M$ as follows. Let $\operatorname{proj}_{T M}(F)=c_{F} \cdot U_{1}$, where $\operatorname{proj}_{T M}$ denotes projection onto $U$ along $T M$. As both the extension of $F$ to $M$ and $U$ are continuous and multivalued, so is $c_{F}$. Then, the modified divergence theorem takes the following form.

Theorem 8.3 (modified divergence theorem). Let $\Omega$ be a region with smooth boundary $\mathcal{B}$ defined by the skeletal structure. Also, let $\Gamma$ be a region in $\Omega$ with regular piecewise smooth boundary. Suppose $F$ is a smooth vector field with discontinuities across $M$, then

$$
\int_{\Gamma} \operatorname{div} F d V=\int_{\partial \Gamma} F \cdot \mathbf{n}_{\Gamma} d S-\int_{\tilde{\Gamma}} c_{F} d M
$$

where $\tilde{\Gamma}$ consists of points $(x, U) \in \tilde{M}$ such that there is an interval $\{x+t U$ : $0<t<\varepsilon\} \subset \Gamma$ for some $\varepsilon>0$.

Remark 8.4. We note that at the edge of $M, U$ becomes tangent, so as we approach the edge $c_{F}$ becomes infinite. However, the integral is still well defined because locally $d M=\rho d S$ and $\rho$ approaches 0 . In fact, as seen in the proof of the theorem, the product $c_{F} \cdot \rho$ represents $F \cdot \mathbf{n}$, for the unit normal vector field $\mathbf{n}$ on $M$, and this remains bounded.

Before proving Theorem 8.3, we derive a consequence for the grassfire flow. We let $G$ denote the unit vector field which generates the grassfire flow. As observed in Example 8.2, $G$ is smooth with discontinuities across $M$. Thus we can apply Theorem 8.3. In this case, $\operatorname{proj}_{T M}\left(-U_{1}\right)=-U_{1}$ so $c_{G}=-1$. Thus, we obtain as a corollary.

Theorem 8.5. If $G$ denotes the unit vector field generating the grassfire flow for the region $\Omega$ with Blum medial axis $M$, then for a piecewise smooth 
region $\Gamma \subset \Omega$

$$
\int_{\Gamma} \operatorname{div} G d V=\int_{\partial \Gamma} G \cdot \mathbf{n}_{\Gamma} d S+\int_{\tilde{\Gamma}} d M .
$$

Remark 8.6. Thus, the flux of the grassfire flow across $\partial \Gamma$ differs from the divergence integral of $G$ over $\Gamma$ by the "medial volume of $\tilde{\Gamma}$ ".

Example 8.7. In the case of $\Omega$ in $\mathbb{R}^{2}, M$ is a branched curve and $\tilde{\Gamma}$ is a union of curve segments in $\tilde{M}$ which represent both sides of the curve segments in $\Gamma \cap M$. The medial measure of $\tilde{\Gamma}$ is twice the integral of $U_{1} \cdot \mathbf{n}$ over $\Gamma \cap M$ with respect to the usual Riemannian length.

Proof of Theorem 8.3. For the proof, we follow the classical proof of replacing the integrals by a sum of local integrals for which the classical divergence theorem is valid. Summing these integrals leads to the modified form in the theorem.

By the properties of skeletal sets we may cover $M$ by the interiors of a finite number of paved neighborhoods $\left\{W_{i}\right\}$. The associated abstact neighborhoods $\left\{\tilde{W}_{i j}\right\}$ are a finite covering of $\tilde{M}$. For each $W_{i}$, we let $\tilde{W}_{i}$, resp., $V_{i}$, denote the union of the $\tilde{W}_{i j}$, resp., radial traces of the $\tilde{W}_{i j}$, for the $\tilde{W}_{i j}$ associated to $W_{i}$. Also, the union of the radial traces of the interiors of the $\tilde{W}_{i j}$ associated to $W_{i}$ form the interior of $V_{i}$ relative to $\Omega$. The unions of the interiors again cover $\Omega$. We let $\left\{\varphi_{i}\right\}$ be a partition of unity subordinate to $\left\{\operatorname{int}\left(V_{i}\right)\right\}$.

We may compute the integral by

$$
\int_{\Gamma} \operatorname{div} F d V=\sum_{i} \int_{\Gamma_{i}} \varphi_{i} \cdot \operatorname{div} F d V
$$

where $\Gamma_{i}=\Gamma \cap V_{i}$. As each $\Gamma_{i}$ is a Borel set, the integrals on the RHS are well defined.

First, we consider the case of a single $F_{i}=\varphi \cdot F$ with support in the interior of a single $V_{i}$. We may represent $V_{i}$ as a union of radial traces $V_{i}=\cup \tilde{M}_{i j \psi}$, where $\pi^{-1}\left(W_{i}\right)=\cup_{j} \tilde{M}_{i j}$ and each $\tilde{M}_{i j}$ is a compact manifold with boundaries and corners with associated smooth value of $U$. Then, we may decompose $\Gamma_{i}$ as a union of $\Gamma_{i j}=\Gamma_{i} \cap M_{i j \psi}$ (figure 11 a). Since $F_{i}$ extends to be smooth on $\Gamma_{i j}$, we may apply the classical divergence theorem.

$$
\int_{\Gamma_{i j}} \operatorname{div} F_{i} d V=\int_{\partial \Gamma_{i j}} F_{i} \cdot \mathbf{n}_{\Gamma} d S .
$$


a)

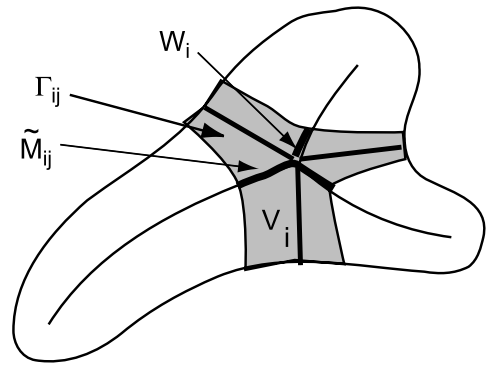

b)

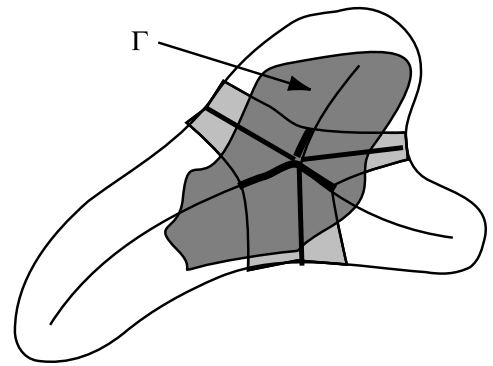

Figure 11: Decomposition of the region $\Gamma$ in $V_{i}$.

Second, we sum (8.4) over $j$. To understand the cancellation that occurs in the sum of the RHS of (8.4), we note as in figure 11b), there are four types of $n$-dimensional faces of $\Gamma_{i j}$ :

(1) common faces of a two distinct $\Gamma_{i j}$;

(2) faces lying in a face of $\partial \Gamma$;

(3) faces lying in a $\tilde{M}_{i j}$ and

(4) faces lying in a radial trace of a boundary facet of $\tilde{M}_{i j}$, not shared with another $\tilde{M}_{i j^{\prime}}$.

For case (1) of common faces of a pair of $\Gamma_{i j}$, the integrals over the faces cancel. For case (4), since the support of $\varphi_{i}$ does not intersect a radial trace of such a single boundary facet, the integral over that face is also 0. For case (2), the sum of integrals over such faces equals $\int_{\partial \Gamma \cap V_{i}} F_{i} \cdot \mathbf{n}_{\Gamma} d S$.

Finally for a face as in case $(3)$, we note that $\mathbf{n}_{\Gamma}=-\mathbf{n}$, the unit normal vector field to $M_{i j}$ pointing in the same direction as the corresponding smooth value of $U$. Also, $F_{i} \cdot \mathbf{n}_{\Gamma}=\varphi_{i}(F \cdot(-\mathbf{n}))$. If we write $F=c_{F} U_{1}+F_{1}$ with $F_{1}$ tangent to $M$, then

$$
\begin{aligned}
F_{i} \cdot \mathbf{n}_{\Gamma} d S & =\varphi_{i} \cdot c_{F}\left(U_{1} \cdot(-\mathbf{n})\right) d S \\
& =-\left(\varphi_{i} \cdot c_{F}\right) \cdot \rho d S
\end{aligned}
$$

where $d S$ the denotes Riemannian volume form on $M$. Then, the sum of the integrals for faces in case (3) equals the integral

$$
\int_{\tilde{\Gamma} \cap \tilde{W}_{i}}-\left(\varphi_{i} \cdot c_{F}\right) \cdot \rho d S=\int_{\tilde{\Gamma} \cap \tilde{W}_{i}}-\left(\varphi_{i} \cdot c_{F}\right) d M .
$$


Hence, summing (8.4) over $j$, we obtain

$$
\int_{\Gamma_{i}} \operatorname{div}\left(\varphi_{i} F\right) d V=\int_{\partial \Gamma \cap V_{i}} \varphi_{i} \cdot\left(F \cdot \mathbf{n}_{\Gamma}\right) d S-\int_{\tilde{\Gamma} \cap \tilde{W}_{i}} \varphi_{i} \cdot c_{F} d M
$$

Equivalently, as $\operatorname{supp}\left(\varphi_{i}\right) \subset V_{i}$, we obtain

$$
\int_{\Gamma} \operatorname{div}\left(\varphi_{i} F\right) d V=\int_{\partial \Gamma} \varphi_{i} \cdot\left(F \cdot \mathbf{n}_{\Gamma}\right) d S-\int_{\tilde{\Gamma}} \varphi_{i} \cdot c_{F} d M
$$

Lastly, we want to sum over $i$. Since $\sum_{i} \varphi_{i} \equiv 1, \sum_{i} \nabla\left(\varphi_{i}\right) \equiv 0$. Then, we may apply a standard argument as follows. Since

$$
\operatorname{div}\left(\varphi_{i} \cdot F\right)=\nabla \varphi_{i} \cdot F+\varphi_{i} \operatorname{div} F
$$

we may sum (8.10) over $i$, using the relations between $\varphi_{i}$ and $\nabla\left(\varphi_{i}\right)$ just stated, to obtain

$$
\sum_{i} \operatorname{div}\left(\varphi_{i} \cdot F\right)=\operatorname{div} F
$$

Thus, summing (8.9) over $i$ using the linearity of the integrals and both (8.10) and (8.11), we obtain

$$
\int_{\Gamma} \operatorname{div} F d V=\int_{\partial \Gamma}\left(F \cdot \mathbf{n}_{\Gamma}\right) d S-\int_{\tilde{\Gamma}} c_{F} d M
$$

which is what we wished to establish.

\section{Computing the average outward flux for the grassfire flow}

We explain in this section how the modified divergence theorem applies to justify an algorithm developed by Siddiqi et al. $[13,15]$ to identify points of the Blum medial axis. Let $\Omega$ be a region with smooth generic boundary $\mathcal{B}$, and Blum medial axis $M$. As in Section 8, we also let $G$ denote the unit vector field which generates the grassfire flow. The algorithm concerns properties of the flux of the vector field $G$, which has discontinuities across $M$.

Suppose we are given a convex region $\Gamma^{\prime} \subset \mathbb{R}^{n+1}$ with regular piecewise smooth boundary, containing 0 in its interior. We can form $t \Gamma^{\prime}=$ $\left\{t \cdot x: x \in \Gamma^{\prime}\right\}$. Then, about any point $x \in \Omega \backslash \mathcal{B}$, we can form the translate 
$\Gamma_{t}(x)=x+t \Gamma^{\prime}$. For $t>0$ sufficiently small, $\Gamma_{t}(x) \subset \Omega$. Thus, we may first consider the limit of the flux across $\partial \Gamma_{t}(x)$ as $t \rightarrow 0$.

$$
\lim _{t \rightarrow 0} \int_{\partial \Gamma_{t}(x)} G \cdot \mathbf{n}_{\partial \Gamma_{t}(x)} d S
$$

where $\mathbf{n}_{\partial \Gamma_{t}(x)}$ denotes the outward normal to $\partial \Gamma_{t}(x)$.

Lemma 9.1. Suppose $\Omega \subset \mathbb{R}^{n+1}$ is a compact region with smooth generic boundary $\mathcal{B}$. If $x \in \Omega \backslash \mathcal{B}$, then

$$
\lim _{t \rightarrow 0} \int_{\partial \Gamma_{t}(x)} G \cdot \mathbf{n}_{\partial \Gamma_{t}(x)} d S=0 .
$$

Proof. If $\Gamma_{t}(x) \cap M=\emptyset$, then by the usual divergence theorem,

$$
\int_{\partial \Gamma_{t}(x)} G \cdot \mathbf{n}_{\partial \Gamma_{t}(x)} d S=\int_{\Gamma_{t}(x)} \operatorname{div} G d V .
$$

By the continuity of $G$ off $M$ and the mean value theorem for integrals,

$$
\int_{\Gamma_{t}(x)} \operatorname{div} G d V=\operatorname{div} G(\bar{x}) \cdot \operatorname{vol}\left(\Gamma_{t}(x)\right)
$$

for some $\bar{x} \in \Gamma_{t}(x)$.

Then, the continuity of $\operatorname{div} G$ implies that $|\operatorname{div} G(\bar{x})|$ is bounded on $\Gamma_{t_{0}}(x)$. If we let $t \rightarrow 0$, then $\operatorname{vol}\left(\Gamma_{t}(x)\right) \rightarrow 0$; hence, the RHS $\rightarrow 0$. Thus, so does the flux.

In fact, we claim this still remains true if $x \in M$. Using the modified divergence theorem applied to $G$ (Theorem 8.5),

$$
\int_{\partial \Gamma_{t}(x)} G \cdot \mathbf{n}_{\partial \Gamma_{t}(x)} d S=\int_{\Gamma_{t}(x)} \operatorname{div} G d V-\int_{\tilde{\Gamma}_{t}(x)} d M .
$$

We claim there is a constant $C>0$ so that for $t>0$ sufficiently small,

$$
\left|\int_{\Gamma_{t}(x)} \operatorname{div} G d V\right| \leq C \cdot \operatorname{Vol}\left(\Gamma_{t}(x)\right) .
$$

If $t$ is small enough, then there is a paved neighborhood $W$ of $x$, so that $\Gamma_{t}(x)$ is contained in the radial trace of $W$. We may then repeat the argument in the proof of Theorem 8.3, to decompose $\Gamma_{t}(x)=\cup_{i} \Gamma_{t i}$, so that each 
$G \mid\left(\Gamma_{t i} \backslash M\right)$ extends smoothly to $\Gamma_{t i} \cap M$. Thus, $\operatorname{div} G$ is bounded on $\Gamma_{t i}$. Thus, there is a single constant $C$ such that

$$
|\operatorname{div} G| \leq C \quad \text { for all } x \in \Gamma_{t}(x)
$$

This continues to hold for all $0<t^{\prime}<t$ as $\Gamma_{t^{\prime}}(x) \subset \Gamma_{t}(x)$. Thus, by again applying the mean value theorem for integrals to each $\Gamma_{t i}$, and then summing, we obtain (9.2).

As $t \rightarrow 0$, the RHS of $(9.2) \rightarrow 0$, so

$$
\lim _{t \rightarrow 0} \int_{\Gamma_{t}(x)} \operatorname{div} G d V=0
$$

Second, since $\rho \leq 1$,

$$
\left|\int_{\tilde{\Gamma}_{t}(x)} d M\right| \leq n-\operatorname{dim} \operatorname{vol}\left(\tilde{\Gamma}_{t}(x)\right)
$$

As $t \rightarrow 0, n$-dim $\operatorname{vol}\left(\tilde{\Gamma}_{t}(x)\right) \rightarrow 0$, so also the second integral on the RHS of $(9.1) \rightarrow 0$ as $t \rightarrow 0$. Thus, as $t \rightarrow 0$, the limit of the flux on $\Gamma_{t}(x) \rightarrow 0$.

Although the limiting value of the flux does not differ for points on or off the Blum medial axis, it turns out that the limiting value of the "average flux" does detect points on the medial axis. The algorithm developed in [13, 15] uses a discrete version of the average flux to determine the Blum medial axis. We justify this algorithm using the modified divergence theorem. In what we say, we restrict attention to regions in $\mathbb{R}^{2}$ or $\mathbb{R}^{3}$, although the general form we give will have analoges in higher dimensions.

We use a convex $\Gamma$ as before, except that we further limit the allowable $\Gamma$ by requiring that the edges of the boundary (where smooth facets meet) lie in the intersection of the boundary with a finite number of hyperplanes passing through 0 . Thus, besides convex regions with smooth boundaries, this also allows, for example, cubes.

We let $\operatorname{vol}_{n+1}\left(\Gamma_{t}(x)\right)$, resp., $\operatorname{vol}_{n}\left(\partial \Gamma_{t}(x)\right)$, denote the $(n+1)$-dimensional volume of $\Gamma_{t}(x)$, resp., $n$-dimensional volume of $\partial \Gamma_{t}(x)$, for $n=1,2$. Now we consider the average flux across $\Gamma_{t}(x)$ to be

$$
\text { the average flux across } \Gamma_{t}(x)=\frac{1}{\operatorname{vol}_{n}\left(\partial \Gamma_{t}(x)\right)} \cdot \int_{\partial \Gamma_{t}(x)} G \cdot \mathbf{n}_{\partial \Gamma_{t}(x)} d S .
$$

We are interested in the limit of the average flux as $t \rightarrow 0$. In particular, we will see that it will again be zero off the medial axis; however, now 
for points on the medial axis, it will not vanish (except at edge points). The non-vanishing on the medial axis is due to the medial integral term in the modified divergence theorem. Thus, we will be concentrating on understanding the contribution of this term to the limiting average flux.

Although the value of the limiting average flux can vary for points on the medial axis, it can be bounded in terms of two invariants associated to each point $x$ on the Blum medial axis $M$. First, we recall that $\rho(x)=U_{1} \cdot \mathbf{n}$ is a piecewise smooth multivalued function on $M$ which has values at $x$ corresponding to each local component of $M$ of $x$, and to each value of $U_{1}$ defined for each local component (and the corresponding unit normal vector $\mathbf{n}$ to the local component at $x$ pointing in the same direction as $\left.U_{1}\right)$. We let $\min (\rho)(x)$ denote the minimum non-zero value of $\rho(x)$ for the multiple values at $x$.

Second, we define for each possible generic type $T$ for points of $M$ a medial density $m_{T \Gamma}$. The generic types for the 1-dimensional medial axis are smooth points, branch points, and end points; for the 2-dimensional medial axis they are smooth points, Y-branch points, edge points, fin points (figure 4a) and 6-junction points (where six local components joined along four Y-branch curves meet). The medial density $m_{T \Gamma}$ has the property that for any point $x \in M$ of type $T$,

$$
m_{T \Gamma} \leq \lim _{t \rightarrow 0} \frac{\operatorname{vol}_{n}\left(\tilde{\Gamma}_{t}(x)\right)}{\operatorname{vol}_{n}\left(\partial \Gamma_{t}(x)\right)}
$$

and this is the largest constant with this property for all generic regions. Here $\operatorname{vol}_{n}\left(\tilde{\Gamma}_{t}(x)\right)$ denotes the integral $\int_{\tilde{\Gamma}_{t}(x)} d V$ for the usual $n$-dimensional Riemannian volume $d V$.

Remark 9.2. We note that this constant can differ for different $\Gamma$ such as a disk versus a cube. In the case of a disk, this gives, up to a constant factor, the local density defined by Kurdyka-Raby for subanalytic sets [14].

We will show that this constant is strictly positive for each type except the edge points of $M$. Then, we define

$$
M_{\Gamma}=\min _{\text {non-edge } T}\left\{m_{T \Gamma}\right\} .
$$

Finally, we will give a bound for the limiting flux at non-edge points of $M$ in terms of $\min (\rho)$ and $M_{\Gamma}$. Since both of these constants are non-zero, we will obtain a non-zero bound for the limiting average flux at non-edge points of $M$. 
The main theorem we will prove is the following.

Theorem 9.3. Suppose $\Omega \subset \mathbb{R}^{n+1}$ for $n=1,2$ denotes a compact region with smooth generic boundary $\mathcal{B}$. If $x \in \Omega \backslash \mathcal{B}$, then

$\lim _{t \rightarrow 0}\left(\right.$ the average flux across $\left.\Gamma_{t}(x)\right)\left\{\begin{array}{cc}=0 & x \notin M \text { or } x \in \partial M \\ <-M_{\Gamma} \cdot \min (\rho)(x) & x \in M \backslash \partial M\end{array}\right.$ where $M_{\Gamma}$ is positive and depends on $\Gamma$ and $\min (\rho)(x)>0$.

Hence, as asserted in [15], the points on the Blum medial axis (except $\partial M)$ are detected by the non-vanishing of the limiting average flux as $t \rightarrow 0$. Another calculation of the limit of the average outward flux in the 1-dimensional case using $\Gamma$ a 2-disk allows a more precise evaluation [24].

Remark 9.4. For a smooth point $x$ of the Blum medial axis, $\rho(x)$ is uniquely defined, and we can give a more precise bound $<-\rho\left(x_{0}\right) \cdot m_{S \Gamma}$ for $S$ denoting the type representing smooth points.

We want to both prove this theorem and to compute these medial densities for the case of disks and cubes. First, we determine how to compute these constants, and do so for $\Gamma$ a 2-disk or square for the 1-dimensional case, or 3-disk or cube for the 2-dimensional case. If we choose a point $x_{0}$ of one type $T$, then we may pave a neighborhood $W$ of $x_{0}$ in $M$ by manifolds with boundaries and corners $M_{i}$. In the 1-dimensional case, these are smooth embedded images of closed intervals, while for the 2-dimensional case, we may take them to be smooth embedded images of closed rectangles.

We let $\tilde{W}$ denote the inverse image of $W$ in $\tilde{M}$, and we let $\tilde{M}_{i j}$ denote the non-edge manifold components of $\tilde{W}$ so each $\tilde{M}_{i j}$ consists of a nonedge manifold component $M_{i}$ of $W$ together with a smooth value of $U$. In practical terms for non-edge points, only in the case of fin points do we disregard a local component (which corresponds to the fin in that case). For each $\tilde{M}_{i j}$, there is a value of $\rho=U_{1} \cdot \mathbf{n}$ at $x_{0}$ which is strictly positive (as $M_{i j}$ is a non-edge component so $U$ is not tangent to $M_{i}$ at $x_{0}$ ). The corresponding function $\rho$ on each $\tilde{M}_{i j}$ is continuous. We let $\tilde{M}_{i j}(t)=\tilde{M}_{i j} \cap \tilde{\Gamma}_{t}\left(x_{0}\right)$. Then,

$$
\int_{\tilde{\Gamma}_{t}\left(x_{0}\right)} d V=\sum_{i, j} \int_{\tilde{M}_{i j}(t)} d V
$$

Thus, to compute

$$
\lim _{t \rightarrow 0} \frac{\operatorname{vol}_{n}\left(\tilde{\Gamma}_{t}\left(x_{0}\right)\right)}{\operatorname{vol}_{n}\left(\partial \Gamma_{t}\left(x_{0}\right)\right)}
$$


it is sufficient to determine for each $\tilde{M}_{i j}$ the limit

$$
\lim _{t \rightarrow 0} \frac{1}{\operatorname{vol}_{n}\left(\partial \Gamma_{t}\left(x_{0}\right)\right)} \cdot \int_{\tilde{M}_{i j}(t)} d V
$$

The $M_{i}$ have well-defined tangent planes at $x_{0}$. Let $R_{i j}(t)$ denote the projection of $M_{i j}(t)$ onto the tangent plane of $M_{i j}$ at $x_{0}$. For $t>0$ sufficiently small, this projection is a diffeomorphism. Also, we let $C_{i}$ denote the intersection in $T_{x_{0}} M_{i}$ of the cone spanned by the half-tangent lines at $x_{0}$ to the 1-dimensional edges of $M_{i}$; and let $C_{i}(t)=\Gamma_{t}\left(x_{0}\right) \cap C_{i}$.

\section{Lemma 9.5.}

$$
\lim _{t \rightarrow 0} \frac{\operatorname{vol}_{n}\left(M_{i j}(t)\right)}{\operatorname{vol}_{n}\left(\partial \Gamma_{t}\left(x_{0}\right)\right)}=\lim _{t \rightarrow 0} \frac{\operatorname{vol}_{n}\left(R_{i j}(t)\right)}{\operatorname{vol}_{n}\left(\partial \Gamma_{t}\left(x_{0}\right)\right)}=\lim _{t \rightarrow 0} \frac{\operatorname{vol}_{n}\left(C_{i}(t)\right)}{\operatorname{vol}_{n}\left(\partial \Gamma_{t}\left(x_{0}\right)\right)} .
$$

Proof of Lemma 9.5. In the 1- or 2-dimensional case (using polar coordinates), a direct calculation shows

$$
\operatorname{vol}_{n}\left(M_{i j}(t)\right), \operatorname{vol}_{n}\left(R_{i j}(t)\right)=\operatorname{vol}_{n}\left(C_{i}(t)\right)+o\left(t^{n}\right)
$$

If we use the scaling property

$$
\operatorname{vol}_{n}\left(\partial \Gamma_{t}\left(x_{0}\right)\right)=t^{n} \cdot \operatorname{vol}_{n}\left(\partial \Gamma\left(x_{0}\right)\right)
$$

together with (9.4), we obtain the result.

Remark 9.6. Almost certainly Lemma 9.5 holds as well for higher dimensions.

Then, applying Lemma 9.5 and (9.3) and keeping in mind that there are two sides $M_{i j}$ to each $M_{i}$, we compute

$$
\lim _{t \rightarrow 0} \frac{\operatorname{vol}_{n}\left(\tilde{\Gamma}_{t}\left(x_{0}\right)\right)}{\operatorname{vol}_{n}\left(\partial \Gamma_{t}\left(x_{0}\right)\right)}=\lim _{t \rightarrow 0}\left(\frac{2}{\operatorname{vol}_{n}\left(\partial \Gamma_{t}\left(x_{0}\right)\right)} \cdot \sum_{i} \operatorname{vol}_{n}\left(C_{i}(t)\right)\right) .
$$

However, by scaling properties of volume for both $C_{i}(t)$ and $\partial \Gamma_{t}\left(x_{0}\right)$,

$$
\begin{aligned}
& \operatorname{vol}_{n}\left(\partial \Gamma_{t}(x)\right)=t^{n} \cdot \operatorname{vol}_{n}(\partial \Gamma) \quad \text { and } \\
& \operatorname{vol}_{n}\left(C_{i}(t)\right)=t^{n} \cdot \operatorname{vol}_{n}\left(C_{i} \cap \Gamma\left(x_{0}\right)\right) .
\end{aligned}
$$


Table 1: Medial densities for the 1-and 2-dimensional cases for $\Gamma$ an $n$-disk or $n$-cube.

\begin{tabular}{|c|c|c|c|c|c|}
\hline \multicolumn{3}{|c|}{ 1-dimensional case } & \multicolumn{3}{|c|}{ 2-dimensional case } \\
\hline Type & 2-disk & Square & Type & 3-disk & Cube \\
\hline \multirow{2}{*}{ Smooth point } & $\underline{2}$ & 1 & \multirow{2}{*}{ Smooth point } & 1 & 1 \\
\hline & $\bar{\pi}$ & $\overline{2}$ & & $\overline{2}$ & $\overline{3}$ \\
\hline \multirow{2}{*}{ Branch point } & 3 & 3 & \multirow{2}{*}{ Y-branch point } & 3 & 1 \\
\hline & $\bar{\pi}$ & $\overline{4}$ & & $\overline{4}$ & $\overline{2}$ \\
\hline \multirow{2}{*}{ End point } & $\underline{1}$ & 1 & \multirow{2}{*}{ Fin point } & 1 & 1 \\
\hline & $\bar{\pi}$ & $\overline{4}$ & & $\overline{2}$ & $\overline{3}$ \\
\hline \multirow{5}{*}{$M_{\Gamma}$} & $\underline{2}$ & $\underline{1}$ & \multirow{2}{*}{ 6-junction point } & $\frac{1}{-}$ & $>1$ \\
\hline & $\pi$ & 2 & & $-\overline{2}$ & 3 \\
\hline & & & End point & $\frac{1}{-}$ & $\frac{1}{a}$ \\
\hline & & & \multirow{2}{*}{$M_{\Gamma}$} & 1 & 1 \\
\hline & & & & $\overline{2}$ & $\overline{3}$ \\
\hline
\end{tabular}

Thus, we conclude from Lemma 9.5 and (9.6)

$$
\lim _{t \rightarrow 0} \frac{\operatorname{vol}_{n}\left(\tilde{\Gamma}_{t}\left(x_{0}\right)\right)}{\operatorname{vol}_{n}\left(\partial \Gamma_{t}\left(x_{0}\right)\right)}=\frac{2}{\operatorname{vol}_{n}\left(\partial \Gamma\left(x_{0}\right)\right)} \cdot\left(\sum_{i} \operatorname{vol}_{n}\left(C_{i} \cap \Gamma\left(x_{0}\right)\right)\right) .
$$

Then, using (9.7) we can compute the medial density.

Proposition 9.7. The medial densities in the 1- and 2-dimensional case for disks and cubes are given by table 1.

Table 1 suggests that for general allowable $\Gamma, M_{\Gamma}=m_{\mathrm{S} \Gamma}$, where $\mathrm{S}$ denotes the type representing smooth points, i.e., the minimum of $m_{T \Gamma}$ occurs for smooth points.

Proof of Proposition 9.7. We consider the 2-dimensional case. The 1-dimensional case is easier. First, for smooth points and fin points $\cup_{i} C_{i}=$ $T_{x_{0}} M$, i.e., in the smooth case $C_{1}=T_{x_{0}} M$, while for a fin point $x_{0}$, there is a unique common limiting tangent plane at $x_{0}$, for each non-edge component and $\cup_{i} C_{i}$ fills out this plane. Thus, the intersection of this plane with a cube $\Gamma$ centered at $x_{0}$ has area $\geq$ the area of a face of the cube. Thus, by (9.7) 
$m_{T \Gamma} \geq 2 \cdot \frac{r^{2}}{6 r^{2}}=\frac{1}{3}$ and equality will occur if the tangent plane is parallel to a face. Instead, for a sphere $m_{T \Gamma}=2 \cdot \frac{\pi r^{2}}{4 \pi r^{2}}=\frac{1}{2}$.

In the case of $Y$-branch points, there are three $C_{i}$ each of which is a half plane. Thus, an analogous computation yields either $\frac{1}{2}$ for a cube or $\frac{3}{4}$ for the sphere.

The remaining case is the 6-junction. There are six cones $C_{i}$ joined along four half-lines through $x_{0}$ which intersect the sphere in four points $P_{i}$. In the case of the sphere or radius $r$ centered at $x_{0}$, the area of each $C_{i} \cap \Gamma\left(x_{0}\right)$ is proportional to the spherical length of the spherical segment determined by $C_{i}$. The sum of the lengths of these 6 segments $>2 \pi r$, and as these points approach a common point, the lengths approach $2 \pi$. Thus, $m_{T \Gamma} \geq 2 \cdot \frac{\pi r^{2}}{4 \pi r^{2}}=\frac{1}{2}$. A similar argument works for the cube giving $\frac{1}{3}$.

Proof of Theorem 9.3. If $x \notin M$, the proof is similar to that for Lemma 9.1. Suppose $|\operatorname{div} G| \leq C$ on $\partial \Gamma_{t}(x)$. By the usual divergence theorem,

$$
\text { average flux across } \begin{aligned}
\partial \Gamma_{t}(x) & =\frac{1}{\operatorname{vol}_{n}\left(\partial \Gamma_{t}(x)\right)} \cdot \int_{\Gamma_{t}(x)} \operatorname{div} G d V \\
& \leq \frac{C}{\operatorname{vol}_{n}\left(\partial \Gamma_{t}(x)\right)} \cdot \operatorname{vol}_{n+1}\left(\Gamma_{t}(x)\right)
\end{aligned}
$$

Using that the volume has the scaling properties (9.6) and

$$
\operatorname{vol}_{n+1}\left(\Gamma_{t}(x)\right)=t^{n+1} \cdot \operatorname{vol}_{n+1}(\Gamma)
$$

we conclude

$$
\text { average flux across } \partial \Gamma_{t}(x) \leq t C \cdot \frac{\operatorname{vol}_{n+1}(\Gamma)}{\operatorname{vol}_{n}(\partial \Gamma)} \text {. }
$$

Since the RHS of $(9.9) \rightarrow 0$ as $t \rightarrow 0$, we conclude that the limit of the average flux is 0 .

For the case of $x \in M$, we again use the modified divergence theorem in the form of Theorem 8.5. We obtain the average flux across $\partial \Gamma_{t}(x)$ by dividing $(9.1)$ by $\operatorname{vol}_{n}\left(\partial \Gamma_{t}(x)\right)$. From $(9.2)$, we obtain

$$
\begin{aligned}
\frac{1}{\operatorname{vol}_{n}\left(\partial \Gamma_{t}(x)\right)} \cdot\left|\int_{\Gamma_{t}(x)} \operatorname{div} G d V\right| & \leq C \cdot \frac{\operatorname{vol}_{n+1}\left(\Gamma_{t}(x)\right)}{\operatorname{vol}_{n}\left(\partial \Gamma_{t}(x)\right)} \\
& \leq t C \cdot \frac{\operatorname{vol}_{n+1}(\Gamma)}{\operatorname{vol}_{n}(\partial \Gamma)}
\end{aligned}
$$


Again as $t \rightarrow 0$, the RHS of $(9.10) \rightarrow 0$. Thus,

(9.11) $\lim _{t \rightarrow 0}\left(\right.$ average flux across $\left.\partial \Gamma_{t}(x)\right)=-\lim _{t \rightarrow 0}\left(\frac{1}{\operatorname{vol}_{n}\left(\partial \Gamma_{t}(x)\right)} \int_{\tilde{\Gamma}_{t}(x)} d M\right)$

It remains to show that the RHS has a non-zero negative value bounded away from zero if $x \in M \backslash \partial M$, while if $x \in \partial M$, the limit is 0 .

Let $x_{0} \in M \backslash \partial M$. We use notation as earlier for a paved neighborhood $W$ of $x_{0}$ with $\tilde{M}_{i j}$ denoting the manifold components of $\tilde{W}$, except we do allow edge-components. We again let $\tilde{M}_{i j}(t)=\tilde{M}_{i j} \cap \tilde{\Gamma}_{t}\left(x_{0}\right)$. Then,

$$
\int_{\tilde{\Gamma}_{t}\left(x_{0}\right)} d M=\sum_{i j} \int_{\tilde{M}_{i j}(t)} \rho_{i j} d V
$$

where $\rho_{i j}$ is the value of $\rho$ for $\tilde{M}_{i j}$. It is enough to determine for each $\tilde{M}_{i j}$ the limit

$$
\lim _{t \rightarrow 0} \frac{1}{\operatorname{vol}_{n}\left(\partial \Gamma_{t}\left(x_{0}\right)\right)} \cdot \int_{\tilde{M}_{i j}(t)} \rho_{i j} d V
$$

Then, for $t>0$ sufficiently small, $M_{i}(t)$ will be connected; thus, we can apply the mean value theorem for integrals to conclude

$$
\int_{\tilde{M}_{i j}(t)} \rho d V=\rho_{i j}(\bar{x}) \cdot \operatorname{vol}_{n}\left(M_{i}(t)\right)
$$

Then,

$$
\lim _{t \rightarrow 0} \frac{1}{\operatorname{vol}_{n}\left(\partial \Gamma_{t}\left(x_{0}\right)\right)} \cdot \int_{\tilde{M}_{i j}(t)} \rho_{i j} d V=\lim _{t \rightarrow 0}\left(\rho_{i j}(\bar{x}) \cdot \frac{\operatorname{vol}_{n}\left(M_{i}(t)\right)}{\operatorname{vol}_{n}\left(\partial \Gamma_{t}\left(x_{0}\right)\right)}\right)
$$

or using $\bar{x} \rightarrow x_{0}$ as $t \rightarrow 0$, Lemma 9.5 , and the scaling properties of volume

$$
\begin{aligned}
& =\rho_{i j}\left(x_{0}\right) \cdot \frac{\operatorname{vol}_{n}\left(C_{i} \cap \Gamma\left(x_{0}\right)\right)}{\operatorname{vol}_{n}\left(\partial \Gamma\left(x_{0}\right)\right)} \\
& \geq \min (\rho)\left(x_{0}\right) \cdot \frac{\operatorname{vol}_{n}\left(C_{i} \cap \Gamma\left(x_{0}\right)\right)}{\operatorname{vol}_{n}\left(\partial \Gamma\left(x_{0}\right)\right)} .
\end{aligned}
$$


Note: if $M_{i}$ is an edge component then $\rho_{i j}\left(x_{0}\right)=0$ so we can drop it from the sum. Summing (9.12) over $(i, j)$ yields

$$
\begin{aligned}
\lim _{t \rightarrow 0}\left(\frac{1}{\operatorname{vol}_{n}\left(\partial \Gamma_{t}(x)\right)} \int_{\tilde{\Gamma}_{t}(x)} d M\right) \geq & \min (\rho)\left(x_{0}\right) \cdot \frac{1}{\operatorname{vol}_{n}\left(\partial \Gamma\left(x_{0}\right)\right)} \\
& \cdot \sum_{i} \operatorname{vol}_{n}\left(C_{i} \cap \Gamma\left(x_{0}\right)\right) \\
\geq & \min (\rho)\left(x_{0}\right) \cdot m_{T \Gamma}
\end{aligned}
$$

by using (9.7), where $x_{0}$ is of type $T$. By (9.11), this is exactly what we claimed

\section{Acknowledgement}

Partially supported by National Science Foundation grants DMS-0405947 and CCR-0310546 and DARPA grant HR0011-05-1-0057

\section{References}

[1] H. Blum and R. Nagel, Shape description using weighted symmetric axis features, Pattern Recog. 10 (1978), 167-180.

[2] S. Pizer, S. Siddiqi, G. Szekely, J. Damon, and S. Zucker, Multiscale Medial Loci and their Properties, Int. J. Comp. Vision 55 (2003), no. 2-3, 155-179.

[3] J. Mather, Distance from a manifold in Euclidean space, Proc. Symp. Pure Math. 40 (1983), (Pt 2), 199-216.

[4] B. B. Kimia, A. Tannenbaum, and S. Zucker, Toward a computational theory of shape: an overview, 'in Three dimensional computer vision', ed. O. Faugeras, M I T Press, 1990.

[5] J. Yomdin, On the local structure of the generic central set, Compositio Mathematica 43 (1981), 225-238.

[6] P. J. Giblin, Symmetry sets and medial axes in two and three dimensions, in 'The Mathematics of Surfaces', eds. Roberto Cipolla and Ralph Martin, Springer-Verlag, 2000, 306-321. 
[7] J. Damon, Smoothness and geometry of boundaries associated to skeletal structures I: sufficient conditions for smoothness, Ann. Inst. Fourier $\mathbf{5 3}$ (2003), 1001-1045.

[8] J. Damon, Smoothness and geometry of boundaries associated to skeletal structures II: Geometry in the Blum case, Compositio Mathematica 140 (2004), no. 6, 1657-1674.

[9] J. Damon, Determining the geometry of boundaries of objects from medial data, Int. J. Comp. Vision 63 (2005), no. 1, 45-64.

[10] H. Weyl, On the volume of tubes, Amer. J. Math. 61 (1939), 461-472.

[11] A. Gray, Tubes, Addison-Wesley Publishers, 1990.

[12] S. Pizer, P. T. Fletcher, S. Joshi, A. Thall, J. Z. Chen, Y. Fridman, D. S. Fritsch, A. G. Gash, J. M. Glotzer, M. R. Jiroutek, C. Lu, K. E. Muller, G. Tracton, P. Yushkevich, and E. L. Chaney, Deformable $M$-reps for $3 D$ medical image segmentation, Int. J. Comp. Vision 55 (2003), no. 2-3, 85-106.

[13] S. Bouix, K. Siddiqi, A. Tannenbaum, and S. Zucker Hamilton-Jacobi skeleton, Intl Conf. Computer Vision, Kerkyra, Greece, (1999) 828-834.

[14] K. Kurdyka and G. Raby Densité des ensembles sous-analytiques, Ann. Inst. Fourier 39 (1989), 753-771.

[15] K. Siddiqi, S. Bouix, A. Tannenbaum, and S. Zucker, The HamiltonJacobi skeleton, Int. J. Comp. Vision 48 (2002), 215-231.

[16] J. Mather, Stratifications and mappings, 'in Dynamical Systems', 'ed. M. Peixoto, Academic Press, New York, 1973.

[17] C. G. Gibson, K. Wirthmuller, A. A. du Plessis, and E. J. N. Looijenga, Topological stability of smooth mappings, Springer Lecture Notes in Math., 552, Springer-Verlag, 1976.

[18] W. Rudin, Real and complex analysis, McGraw-Hill, New York, 1966.

[19] M. Spivak, A comprehensive introduction to differential geometry, $\mathbf{I}-\mathbf{V}$, Publish or Perish Inc., Berkeley, CA, 1975, 1979.

[20] A. Lieutier, Any open bounded subset of $\mathbb{R}^{n}$ has the same homotopy type as its medial axis, Proc. 8th ACM Sympos. Solid Modeling and Appl., 2003,65 .

[21] M. Greenberg, Lectures on algebraic topology, W. A. Benjamin, 1967. 
[22] G. Stetten Volume from boundary curvature and medial scale, Technical report UNC Department of Computer Science, 1998.

[23] L. Loomis and S. Sternberg, Advanced Calculus, revised edit., Jones and Barlett Publishers, Boston, MA, 1990.

[24] P. Dimitrov, J. Damon, and K. Siddiqi, Flux invariants for shape, Proc. IEEE CVPR, 2003, 1063-1069.

[25] J. W. Bruce, P. J. Giblin, and C. G. Gibson, Symmetry sets, Proc. Roy. Soc. Edinburgh 101A (1983), 163-186.

[26] M. Goresky, Triangulation of stratified objects, Proc. Amer. Math. Soc. 72 (1978), 193-200

[27] J. Munkres, Elementary differential topology, Annals of Math. Studies, 54, Princeton University Press, Princeton, 1961.

[28] J. Verona, Stratified mappings - structure and triangulability, Springer Lecture Notes 1102, Springer-Verlag, 1984.

Department of Mathematics

University of North CAROLINA

Chapel Hill

NC 27599-3250

USA

E-mail address: jndamon@math.unc.edu

RECEIVED July 10, 2005 